\title{
EL FINANCIAMIENTO DE LA SALUD EN ECUADOR
}

\section{FUNDING HEALTH IN ECUADOR}

RUTH LUCIO ${ }^{1}$

RODRIGO LÓPEZ ${ }^{2}$

NICOLE LEINES 3

JUAN ANTONIO TERÁN ${ }^{4}$

Recibido: 12 de abril

Aceptado: 1 de mayo

\footnotetext{
${ }_{1}^{1}$ PhD (Doctorado Programa Epidemiología y Salud Pública), Universidad de Alcalá UAH, ruthlucior@gmail.com, +593993344968.

${ }^{2}$ Economista, Universidad San Francisco de Quito. MSc. Econometrics and Economics, The University of York, rodrigo.lopezsantos@gmail.com

${ }^{3}$ Economista, Pontificia Universidad Católica del Ecuador, leinesnicole@gmail.com

${ }^{4}$ Matemático Louisiana State University Shreveport (LSUS), juanantonioteran@icloud.com
} 



\title{
EL FINANCIAMIENTO DE LA SALUD EN ECUADOR
}

\section{FUNDING HEALTH IN ECUADOR}

\author{
Ruth Lucio, Rodrigo López, Nicole Leines, Juan Antonio Terán
}

Palabras clave: Derechos, Salud, Gasto en Salud, Seguro de Salud, Programas de Subsidios Médicos, Gasto en Salud del Gobierno Nacional, Salud Pública, Regulación

Keywords: Entitlements, Health, Health Expenditure, Health Insurance, Medical Subsidy Programs, National Government Health Expenditure, Public Health, Regulation

\section{RESUMEN}

El artículo explora la importancia y alcance que tienen las actividades, recursos y productos de salud en la economía ecuatoriana en la actualidad, así como las necesidades de financiamiento sectoriales para sostener un modelo de Sistema Nacional de Salud definido constitucionalmente de manera bicéfala. Para ello, además de establecer las cifras de los agregados con una visión sistémica, aborda aspectos concretos sobre la estructura del talento humano y del impacto económico que conlleva la dotación de medicamentos de alto costo en la dinámica financiera del Gobierno. Concluye con recomendaciones sobre las medidas a implementar para que el financiamiento del sistema de salud ecuatoriano no colapse en breve. 


\section{SUMMARY}

The article explores the importance and scope of activities, resources and health products in the Ecuadorian economy at present, as well as the needs of sectoral financing to sustain the National Health System Model, constitutionally defined in a two-sided manner. To do this, in addition to establishing aggregate figures with a systemic vi- sion, it addresses specific aspects of the structure of human resources and the economic impact of the provision of high-cost drugs in Government's financial dynamics. It concludes with recommendations on specific measures to be implemented, so that, financing of the Ecuadorian health system does not collapse in the short-run.

\section{ELEMENTOS SOBRE EL FINANCIAMIENTO DE LA SALUD EN ECUADOR}

\section{Introducción}

El financiamiento de la salud, circunscrito mayormente a servicios de salud, es un tema que empieza a visualizarse como un problema para Ecuador, a medida que incrementa la población, el porcentaje de población adulta-mayor empieza a crecer a tasas más elevadas que la de niños y jóvenes, y las tecnologías sanitarias — como medicamentos o dispositivos médicos - se comercializan a precios exorbitantes, elementos todos que implican un fuerte impacto financiero para cualquier presupuesto existente. Coadyuva también a esta situación, el reconocimiento constitucional del acceso a servicios de salud como un derecho humano de segunda generación y con ello que el Gobierno tenga obligación de contribuir de manera importante en la sostenibilidad financiera del sistema de salud, para garantizar el acceso universal y gratuito a servicios de salud para la población que habita en Ecuador.

El financiamiento sin embargo, se transforma en un real problema cuando su alcance y comportamiento no es fácilmente aprehensible y por tanto manejable, lo cual en el país ocurre por tres razones principales: 1) Existencia de múltiples agentes, cuya incidencia financiera no es clara, asequible, confiable ni homologada (oferta) e inexistencia de una base nominalizada país para atención médica (demanda), lo cual dificulta captar el alcance del sector en referencia al PIB. 2) Desinterés por parte de los agentes financieros por transparentar 
su financiamiento, lo cual genera incertidumbre sobre la cobertura y alcance real del financiamiento en salud del país. 3) Inexistencia de una instancia con un rol especializado específico de control financiero de las diferentes instancias que manejan recursos para adquirir la provisión de servicios de salud.

El objetivo del estudio consiste en explorar la importancia y alcance que tienen las actividades, recursos y productos de salud, así como las necesidades de financiamiento para sostener el Sistema Nacional de Salud, así como también el establecimiento de cifras de los agregados con una visión sistémica, detectando problemas estructurales y abordando aspectos concretos sobre la estructura del talento humano y del impacto económico que conlleva la dotación de medicamentos de alto costo.

El presente estudio se trata de un análisis descriptivo de carácter sectorial, para lo cual se realizó una revisión documental de registros administrativos, informes y encuestas de carácter nacional e internacional, sobre el sector salud en el último lustro. Adicionalmente se procesaron bases de datos sectoriales en ámbitos demográficos, económicos, financieros, distributivos-remunerativos y presupuestarios, con una representación a nivel nacional y en casos específicos, institucional.

Este artículo inicia con una revisión de la literatura académica sobre el financiamiento en la salud, generando un contexto teórico en el que inscribe el análisis funcional del Sistema Nacional de Salud ecuatoriano.

El estudio explora la importancia y alcance financiero que tienen las actividades y productos de salud en la economía ecuatoriana en la actualidad, para lo cual se analiza información demográfica y económica sobre el gasto en salud. Comparando la economía ecuatoriana con el resto de países de la región, se estima el valor per cápita invertido en salud y se contrasta el resultado financiero de Ecuador versus el de los países latinoamericanos.

Posteriormente, se identifica el alcance y peso que tienen las actividades de salud en la economía ecuatoriana, para lo cual se analiza la composición del financiamiento profundizando sobre el rol de los principales actores financiadores de los servicios de salud y definiendo los roles institucionales asumidos en las dimensiones del financiamiento.

En la siguiente sección se analiza la oferta y cobertura de los diferentes subsistemas, evidenciando que la gran cobertura pública prácticamente ha llegado a la universalidad. Sin embargo, llama la atención que el rol financiador del Gobierno tiende a cambiar su dinámica creciente, enfatizándose un mayor protagonismo de los hogares en la financiación de la salud, sobre todo a través 
de su contribución a aseguramientos públicos.

Finalmente, se analiza la incidencia económica global de componentes como el talento humano y medicamentos, que son claves dentro del financiamiento del sector salud.

\section{Referentes teóricos}

Los estudios sobre financiamiento en salud, aunque limitados en número, presentan distintas dimensiones para su análisis. Estos van desde una visión eminentemente descriptiva sobre la organización y composición de los sistemas, los grupos poblacionales cubiertos por cada subsistema, las prestaciones y características del acceso efectivo, las fuentes de financiamiento, el valory mecanismos de transferencia; hasta la minucia del gasto efectuado y su impacto en las diferentes economías.

En el "Informe sobre la salud en el mundo 2000 - Mejorar el desempeño en los sistemas de salud" (WHO, 2000) se establece a un sistema de salud como "todas las actividades cuya finalidad principal es promover, restablecer o mantener la salud", teniendo tres principales objetivos: (i) mejorar la salud de la población; (ii) responder a las expectativas de las personas; (iii) brindar protección financiera contra los costos de enfermedad. Con ello, el análisis de los mecanismos de aseguramiento y protección financiera en salud resultan centrales y se establecen como una línea deseable de investigación (Maceira, 2014). Por ello la producción de datos sobre este particular se empieza a generar de fuentes estandarizadas de datos para lograr la comparación de los sistemas de salud entre países y su caracterización bajo similares parámetros. Publicaciones de este tipo se encuentran en los denominados perfiles sanitarios que OPS produce para países de la Región, Ecuador incluido.

Posteriormente en el primer lustro de los noventa, el desarrollo de la literatura refiere a la organización y financiamiento de los sistemas de salud. Los indicadores sirven como fuente de información para la elaboración de matrices comparativas entre grupos. Dentro de este bloque, el análisis sistémico de los modelos de salud en América Latina, provee resultados comparados sobre gasto en salud y su composición entre subsectores.(OMS, n.d.). A decir de Maceira, fundamentalmente estos estudios permitieron abrir el debate metodológico sobre factores relevantes para la determinación de la incidencia del gasto público sobre el total de las erogaciones en salud, el peso relativo de las instituciones de seguridad social y el papel desempeñado por el gasto de bolsillo (Maceira, 2014). En dicho período y paralelamente, se habría iniciado tam- 
bién el desarrollo de una línea de trabajo desde la microeconomía, vinculada con organización, incentivos institucionales y desempeño relativo de los sistemas de salud, con estudios comparados a nivel regional latinoamericano.

La emergencia de encuestas de similar formato en varios países, así como los estudios de Cuentas Nacionales en Salud, abren a inicios del 2000 varias líneas de trabajo que analizan fundamentalmente el comportamiento del gasto en salud de las familias y el impacto progresivo o regresivo del mismo dentro del ingreso familiar y de la economía en su conjunto. Aquí se brinda las bases para establecer mecanismos de protección social de las familias ante shocks de salud. Es importante resaltar que a partir de esto y hasta la actualidad se empiezan a diseñar metodologías e indicadores de medición uniforme entre países, y de donde surgen conceptos tales como umbrales de pobreza y gasto empobrecedor, entre otros (Sojo, 2017).

Durante los años 2000 -y Ecuador no escapa de esta tónica - emerge en los países latinoamericanos un cuerpo de literatura en sistemas comparados de salud asociado con reformas, que contemplan temáticas variadas vinculadas con evaluaciones puntuales de reformas financieras, tendencias en reformas y ejes tradicionales (separación de funciones, descentralización, entre otros); y la economía política de los cambios en sistemas de salud (Maceira, 2014). En este contexto se vuelve importante el rol del Estado en la salud y la definición sistémica bajo la cual se organizan los sistemas. Temas centrales como la articulación del financiamiento, la dilución del riesgo de los gastos en salud, la segmentación/ fragmentación de los sistemas, la gobernanza y mecanismos de pago acordes a una distribución eficiente y equitativa; empiezan a ser la tónica de los análisis (Giovanella, L, Feo, O, Faria, M, Tobar, 2012; OPS/OMS, 2017)(Miguel \& Malo, 2014).

Los años 2000 también marcarán la emergencia de investigaciones bajo marcos organizativos funcionales para las reformas, y no por grupos sociales, para asegurar los derechos a la población, emergen trabajos académicos como el de Londoño y Frenk que ponen la tónica hasta el presente (Londoño \& Frenk, 1997). A fines de la década en 2008 Kutzin realiza una publicación en la cual sintetiza a las funciones del sistema de salud en: generación de recursos (recaudación), acopio (pooling de riesgo), distribución y compra, y entrega de los servicios; transformando a objetivos intermedios — de las políticas en saluda la calidad, la equidad en la utilización de recursos, eficiencia, transparencia y responsabilidad (accountability), (Kutzin, 2008)grounded in the core values es- 
poused by WHO; (2. Por lo cual al evaluar el desempeño sistémico deja de lado aquellas que reflejan de mejor manera el estatus de salud, la satisfacción del ciudadano y la protección contra el riesgo financiero (Maceira, 2014).

Para el caso de Ecuador entre los principales análisis se cuenta con la descripción del sistema de salud de Ecuador, donde los autores hacen una descripción de las condiciones de salud en el país y se adentran en las características funcionales del sistema, incluyendo los aspectos de financiamiento (Lucio, Villacrés, \& Henríquez, 2011)(Carriel-Mancilla, 2012).

En la última década la literatura plantea marcos específicos relacionados con el acceso y cobertura universal de salud (OMS, 2010; OPS/OMS, 2014, 2015) y la generación de estrategias específicas como la ampliación del espacio fiscal para la salud (OPS/OMS, 2018), bajo un aprendizaje en el cual la discusión no es más cuál sistema impulsar, sino cómo lograr una racionalidad única en las decisiones financieras, para que las diferentes fuentes de ingresos no sean sustitutivas sino complementarias.

Para efectuar este análisis descriptivo y transversal, donde se analiza la política pública en salud y sus resultados para la economía ecuatoriana, partimos del marco teórico creado por Joseph Kutzin (OMS), donde realiza la definición de las dimensiones funcionales del financiamiento, tales como: recaudación, aseguramiento o administración y pago a proveedores; así se organiza el financiamiento desde los diferentes actores y el rol que desempeñan en cada dimensión. Este marco conceptual nos permite reconocer en cada dimensión a quiénes son los actores fundamentales, así desde la dimensión recaudatoria se identifica en dónde se generan los recursos para fondear los servicios de salud, principalmente los hogares y el gobierno, desde la dimensión aseguradora quiénes administran los recursos y desde la provisión, quiénes están captando los recursos y en qué magnitud. Ninguno de estos roles podría ser aprehendido desde una perspectiva cotidiana mucho más cuanto, si bien analíticamente la diferencia es nítida, en los hechos la mayoría de actores en los sistemas sanitarios, conllevan intrínsecamente más de una figura.

Adicionalmente al enfoque funcional mencionado, se toma el marco conceptual de la Organización Mundial de la Salud, donde se establecen las preguntas que definen el alcance del financiamiento y que consideran los conceptos sobre cobertura poblacional, cobertura prestacional, protección financiera y calidad de las prestaciones, que utilizamos en este análisis. Este es clave para formular las preguntas específicas a contestarse en un análisis de esta naturaleza, por lo cual 
constituye cada una de ellas un referente en el desarrollo de este artículo.

En el análisis se maneja una visión teórica que inscribe el análisis funcional dentro de un marco sistémico, en donde el conjunto de elementos o subsistemas están interrelacionados con el objetivo común de brindar atención de salud a toda la población. Al estar todos los elementos - o subsistemas - relacionados se alcanza el nivel de resultados que esperamos cuando el sistema empieza a funcionar. Deseable alcanzar la cobertura total, en los plazos requeridos, dentro de los presupuestos manejados y de acuerdo a parámetros de riesgo aceptables.

Finalmente, para el análisis del financiamiento de la salud en Ecuador no es posible obviar la teoría del gasto público, en particular cuando se considera a la salud como un bien público y el Estado tiene un rol particular en el suministro y producción de servicios de salud. Sin embargo, en este enfoque subyace todo el análisis anterior que se especifica en una medida o ámbito determinado.

\section{Crecimiento económico e inversión en salud en la economía ecuatoriana}

El crecimiento de la economía ecuatoriana marca el ritmo de los resultados obtenidos en la última década, de esto se deriva un incremento del Producto Interno Bruto, una mayor inversión sanitaria y mejores indicadores en salud; que sin embargo, siguen siendo un desafío en el presente y futuro, como se observa seguidamente.

\section{a. Producto Interno Bruto y Demo- grafía}

La población que habita en Ecuador se estima en 17,2 millones de personas. En la década 2010 - 2020 la tendencia demográfica señala una tasa de decrecimiento en el grupo de niños y adolescentes y simultáneamente una tasa de crecimiento sostenida en la proporción de los adultos mayores, evidenciando que la ventana de oportunidad ${ }^{1}$ se restringe de manera importante para el país, justo a fines de la década (INEC, 2015).

\footnotetext{
${ }^{1}$ En términos demográficos se considera como Ventana de oportunidad, a aquel período en el cual la población joven es todavía proporcionalmente más grande que la adulta mayor.
} 


\section{Gráfico 1. Ecuador: Tendencia relativa de grupos etáreos seleccionados} (2010-2020)

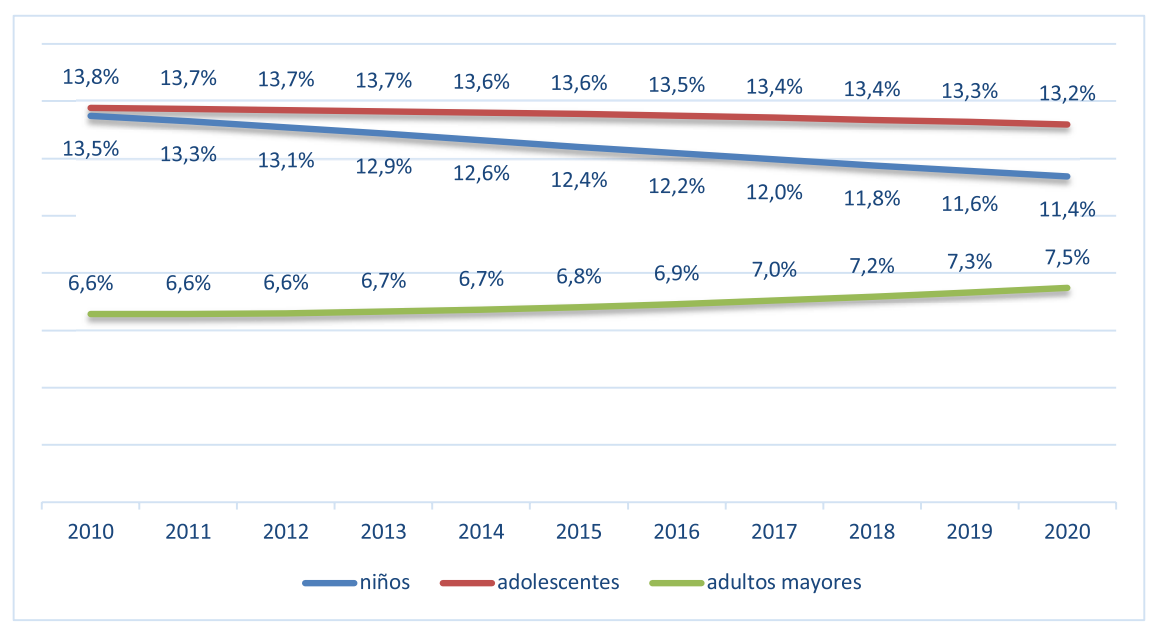

Fuente: Proyecciones de población Nacional y grupos de edad simple. INEC (2015)

La riqueza generada en el país América Latina, como Chile y Guatemadurante 2019, por la dinámica de su población, se estima alcanzará un Producto Interno Bruto de USD 113 mil millones de dólares corrientes. Si comparamos cifras con países que tienen similares tamaños poblacionales en la, para 2017 la producción ecuatoriana representa respecto del primero alrededor de un tercio del PIB, mientras para el segundo caso, Ecuador observa un mejor desempeño con una producción superior en un $27 \%$. 


\section{Gráfico 2. PIB global y per cápita comparado con países latinoamericanos con tamaño poblacional similar}

(2017)

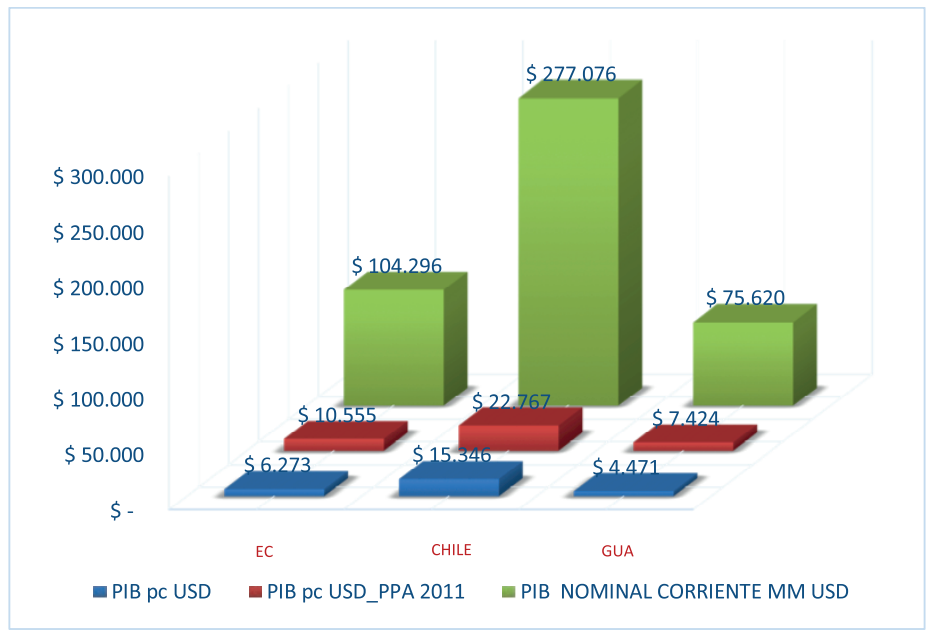

Fuente: (1) población 2017. BM; (2) pib pc,ppa,total 2017 BM

De todas formas, y aunque ha yecciones de crecimiento inexistentes o crecido consistentemente, para 2017 modestas de alrededor del-0,5\% (BM) o Ecuador con un per cápita de USD 6.273 0,7\% para el 2019 (FMI), lo cual de conse encuentra todavía muy por debajo del cretarse sin duda dificultará aún más su promedio del PIB per cápita regional de posicionamiento regional. USD 9.163. Se suman a lo anterior pro- 


\section{Gráfico 3. Ecuador: Producto Interno Bruto Per Cápita comparado con países de América Latina \\ (USD 2017)}

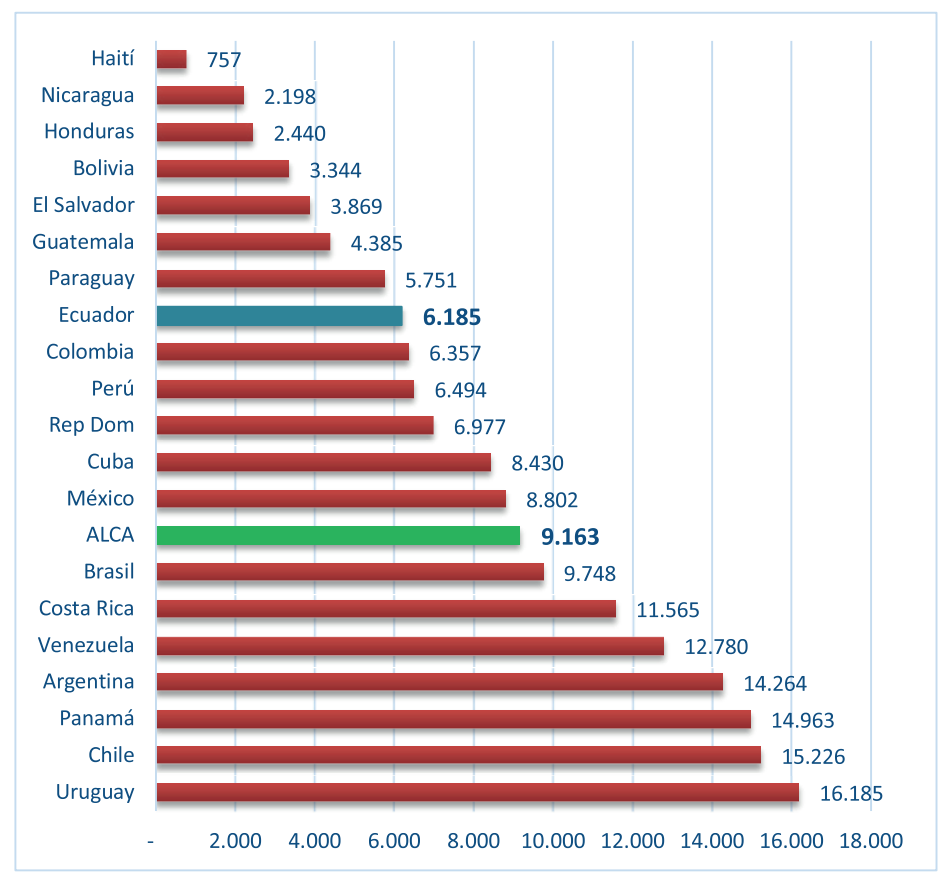

Fuente: BM

Por otro lado, observar comparativamente con países de la región, la ubicación cartesiana de coordenadas formadas por el porcentaje del PIB (X) y logros sanitarios estratégicos obtenidos, como la Razón de Mortalidad Materna $(Y)$, nos permite situarnos en la creciente importancia de la relación óptima entre la riqueza alcanzada por un país y los logros alcanzados en salud. Si tomamos como referencia el promedio regional $(287,59)$ para el año 2017 encontramos que Ecuador, ubicado en el punto $(104,40)$, se encuentra entre los países que tienen un resultado sanitario mejor que la media de 59 muertes maternas $x$ 100.000 nacidos vivos, con una producción o riqueza bastante menor al prome- 
dio regional de 104 a 288 mil millones de dólares. Esto evidencia para Ecuador un resultado sanitario importante en medio de una producción limitada, a la par de una lejanía frente a los referentes Mexicano, Brasileño y por supuesto al canadiense con tan solo seis muertes maternas x 100.000 NV y un PIB de 1,65 mil millones. Importante entender que no siempre una mayor riqueza significa un mejor resultado sanitario, por lo cual el seguimiento a la calidad del gasto es imperativo.

\section{Gráfico 4. PIB y Razón de Mortalidad Materna en América Latina (USD Miles Millones 2017)}

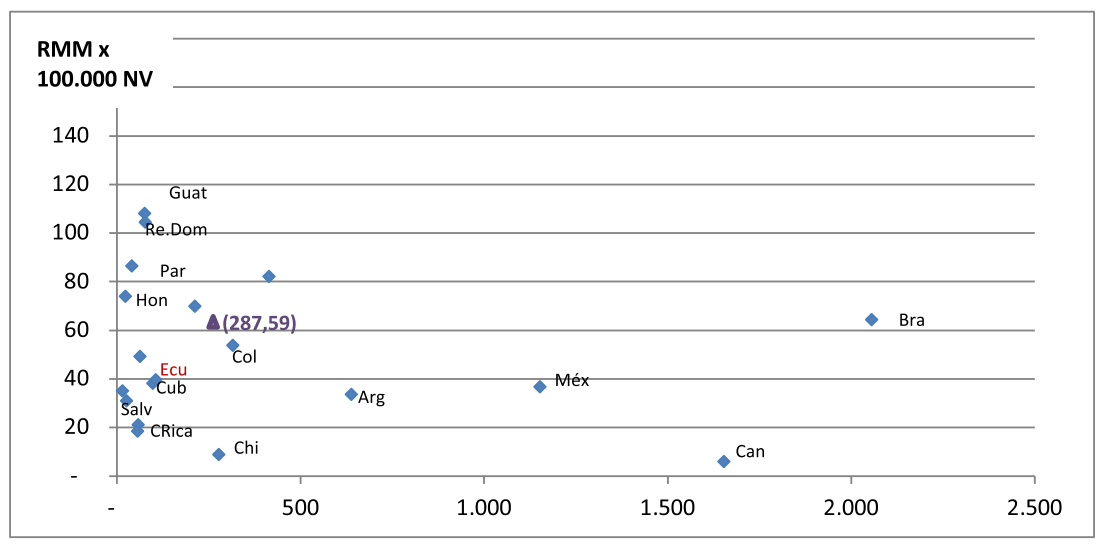

Fuente: (OMS, n.d.)

En el contexto mencionado, existe la tesis de que invertir en salud marca la diferencia entre países desarrollados y no desarrollados. De hecho, los índices comparativos de la última década han demostrado una asociación positiva directa entre salud y desarrollo. Esto es resultado de al menos dos factores: me- joramiento de las condiciones y capacidades del capital humano y generación de una dinámica económica con ventajas comparativas, concepto que a su vez nos induce a observar el detalle de la inversión financiera en salud del Ecuador (Xu K, Saksena P, Jowett M, Indikadahena C, Kutzin J, 2010). 


\section{b. Relación entre Economía y Salud}

En el contexto mencionado es importante observar que salud representa el 8\% del PIB en Ecuador a 2016 (OMS, n.d.). Dicho porcentaje no es menor, ya que las economías desarrolladas se manejan en un rango promedio del $9 \%{ }^{2}$

La producción de riqueza del sector salud se expresa, entre otros aspectos, en que constituye una fuente de empleo directa para al menos 205 mil jefes de familias (cita). Si se estima que por cada empleo directo en salud se generarían 2,04 empleos indirectos adicionales ${ }^{3}$, estamos hablando de alrededor de 420 mil personas (Durán-Lima J, 2016), lo cual representa poco más del $5 \%$ de la Población Económicamente Activa (PEA) en Ecuador a 2018, y alrededor del 12,5\% de la población con empleo adecuado, característico de este sector (INEC, 2018).

Si entendemos a la Salud como el estado de completo bienestar físico, mental y social, y no solamente la ausencia de afecciones o enfermedades; podemos entenderlo fácilmente desde la perspectiva económica como un sector intrínsecamente dinámico debido a la amplitud en el rango de producir bienes y servicios de la más diversa índole que en su mayoría están orientados a restituir el bienestar físico de las personas. Estos abordan ámbitos de infraestructura, equipamiento, medicamentos, biológicos, reactivos, insumos, ropa blanca-uniformes, vehículos, prótesis-férulas, aditamentos para funcionalidad, física, motora, visual, auditiva, bancos de productos biológicos, entre los más comunes, hasta servicios formativos de talento humano diverso, realización de pruebas de laboratorios e imágenes diagnósticas, servicios de rehabilitación, cuidados paliativos, trasplantes, investigación científica en salud; así como contratación o presencia de profesionales para brindar la atención sanitaria tanto de carácter público como privado. Por sus características, las actividades de salud aportan al crecimiento de otros sectores conexos como aquellos importadores y exportadores, industriales, educativos, de la construcción, comerciales, de transporte y aquellos de investigación de alta tecnología. Se estima que entre 2007 y 2014 de cada USD 100 de riqueza generada en el proceso de producción únicamente del servicio de salud, USD 34 fue generado por el sector privado ecuatoriano y 66 por el sector público (BCE, n.d.).

\footnotetext{
${ }^{2}$ EE.UU. (17,07\%), Canadá (10,5\%), UK (9,8\%), Francia (11,5\%), Suiza (12,2\%).

${ }^{3}$ Si bien esta cifra puede parecer muy baja, puesto que usualmente la literatura estima como un estándar la generación de cinco empleos indirectos por uno directo, el estudio de Durán Lima y Castresana, permite realizar inferencias sectoriales más precisas, que de acuerdo a estimaciones propias resultarían en que para salud las cifras son de 1 directo por 2,04 indirectos.
} 
Es claro que disminuir el gasto en salud impacta en la reducción de la esperanza de vida de las poblaciones, teniendo como consecuencia una directa disminución de la tasa de crecimiento económico, resultado de la pérdida de productividad de la fuerza de trabajo. Estudios realizados determinan por ejemplo que, como consecuencia de una disminución en la esperanza de vida del $40 \%$, debido a un recorte presupuestario en salud, la tasa de crecimiento económico disminuiría en 1,4 puntos porcentuales por año (Barro, 2013).

Por fortuna una de las razones por las cuales el país ostenta una cifra importante del gasto/inversión en salud respecto al PIB, se debe al hecho de que en Ecuador se ha definido la vigencia de un modelo de salud universal, desde hace por lo menos la última década.

\section{Gráfico 5. Dimensiones del Acceso y Cobertura Universal en Salud}

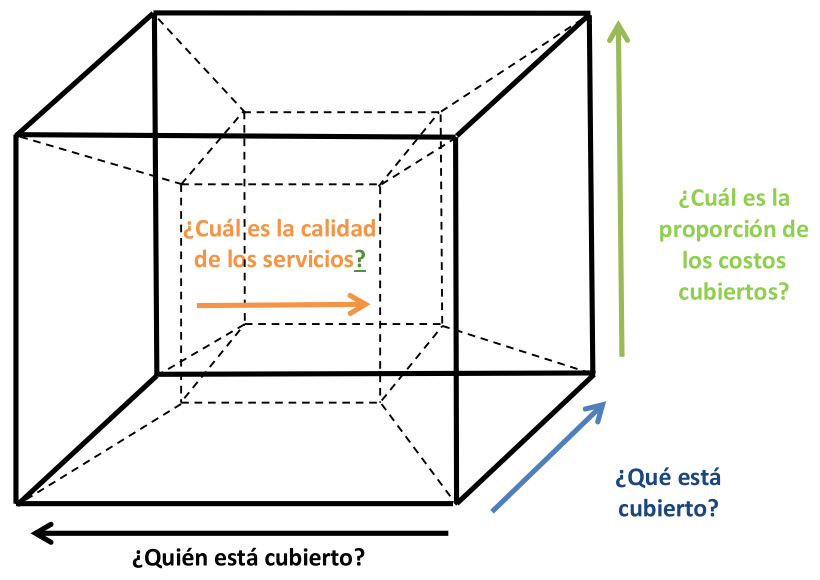

Fuente: 0MS, 2010

En vigor desde el siglo pasado, el modelo fue constitucionalmente refrendado en el año 2008, y ha venido construyéndose bajo la premisa de garantizar el acceso universal en Ecuador en una cuádruple dimensión de totalidad: Todos los habitantes, Todas las prestaciones, Protección financiera y Calidad de la atención. Así en Ecuador todos sus habitantes tienen derecho a acceder a ser- 
vicios de salud integrales, integrados y continuos ${ }^{4}$. (Ministerio de Salud Pública. Subsecretaría Nacional de Gobernanza de la Salud Pública, 2013).

La Salud es un bien público por excelencia, financiarla significa destinar consistente e inteligentemente recursos económicos para solventar las cuatro dimensiones del sector (Stiglitz, 2003). Esto implica que un porcentaje creciente de la captación de recursos deberán ser destinados al uso específico de fomentar hábitos saludables, prevenir riesgos que afecten la salud, preservar y curar la salud de la población, proveerle de cuidados paliativos y acompañarla en el buen morir. La literatura científica menciona que uno de los canales de influencia de la salud sobre el crecimiento económico es la reducción de las tasas de mortalidad y enfermedad y por lo tanto, la disminución de la tasa efectiva de depreciación del capital humano se ralentiza, impactando directamente en el crecimiento económico.

Con lo previamente mencionado como fundamentos conceptuales, el gobierno ecuatoriano para 2016 aportó tan solo con aproximadamente el $51 \%$ del financiamiento de la salud en el país, cifra que indica que todavía un amplio margen es sustentado por los hogares. Adicionalmente comparado con los países latinoamericanos, queda por debajo de la mayoría de aquellos, en su participación.

\footnotetext{
${ }^{4}$ Integralidad se refiere a desarrollar acciones integrales de atención orientadas a la promoción, prevención, curación, rehabilitación y cuidados paliativos, para la satisfacción de las usuarias y usuarios (de manera integrada y continua) en los tres niveles de atención de la red de servicios de salud.
} 


\section{Gráfico 6. Gasto del Gobierno como porcentaje del Gasto total en salud} (2016)

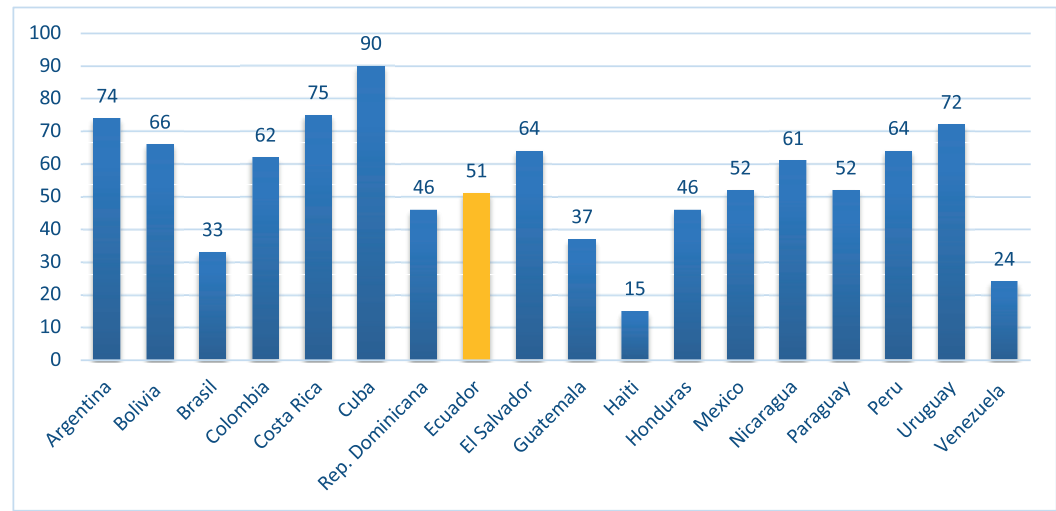

Fuente: (OMS, n.d.) consultado 05.marzo 2019

Para adentrarse a establecer con respecto al Producto Interno Bruto una relación entre economía y salud en generado. Allí encontramos que este Ecuador, dada la presencia fundamental porcentaje va disminuyendo progresidel financiamiento gubernamental, es vamente y se esperaría para 2019 una necesario analizar la participación del participación menor a la de 2016, en alPresupuesto General de Estado (PGE) rededor de 2,7\%.

Tabla 1. Participación porcentual del Presupuesto General del Estado con respecto al PIB

(2016-2019)

\begin{tabular}{|c|c|}
\hline Año & \% PGE/PIB \\
\hline 2016 & $34,1 \%$ \\
\hline 2017 & $33,3 \%$ \\
\hline 2018 & $31,8 \%$ \\
\hline 2019 & $31,4 \%$ \\
\hline
\end{tabular}

Fuente: BCE valores corrientes, el PIB del año 2016 y 2017 es semidefinitivo y provisional respectivamente. Información tomada de las Cuentas Nacionales Anuales; el PIB del año 2018 y 2019 es tomado de Previsiones Macroeconómicas 2018 
El déficit estimado para 2019 en USD 3.655 millones, equivalente a 3,2\% del PIB, y las necesidades de financiamiento por USD 8.166 millones, aunadas a las expectativas de restricciones económicas en los hogares ${ }^{5}$, fuente principal de los impuestos que financian sobre el $90 \%$ de los ingresos del Gobierno Nacional, colocan las finanzas públicas en un momento de limitaciones incuestionables.

Lo anterior puede ser fácil de comprender si se conoce que también en 2018 en Ecuador, y de forma atípica en el mundo, se asume constitucionalmente de forma simultánea, la universalización del sistema de seguridad social en salud también, con lo cual se adquiere la obligatoriedad de financiar también dos modelos de financiamiento universales, desde las fuentes existentes. Ello origina que, adicional a mantener un Sistema Nacional de Salud basado en impuestos (Beveridge), también se mantiene un Sistema de Aseguramiento Universal basado en contribuciones
(Bismarckiano). Entre muchas consecuencias esto hace que, por un lado los hogares ecuatorianos aporten para el sistema público de salud, a través de, por ejemplo, el impuesto a la renta o al valor agregado, mientras por otro lado los mismos hogares aporten a través de contribuciones obligatorias o voluntarias de sus ingresos.

Si observamos el promedio del gasto sanitario público en salud en Ecuador, que para efectos comparativos incluye también la seguridad social pública, este rubro alcanza para 2016 el $4,4 \%$ con respecto al PIB. Comparado con el 3,8\%, el promedio de la región en dicho año es significativo, aunque se queda corto frente a la recomendación de la OPS/OMS, de destinar al menos un $6 \%$ del PIB.

Para 2019 se presupuesta en Ecuador que el gasto sanitario público alcanzará el 5,2\% con respecto al PIB. Si observamos únicamente el financiamiento del Ministerio de Salud Pública, este llegará solamente el 3,1\% del PIB.

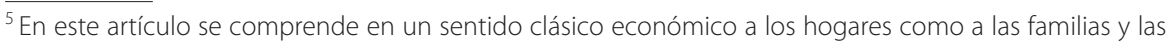
empresas de una sociedad.
} 


\section{Gráfico 7. Presupuestos del Estado (PGE), Subsistemas Públicos de Salud (RPIS) y Sectorial del Ministerio de Salud Pública (MSP) \\ (Miles de millones de USD)}

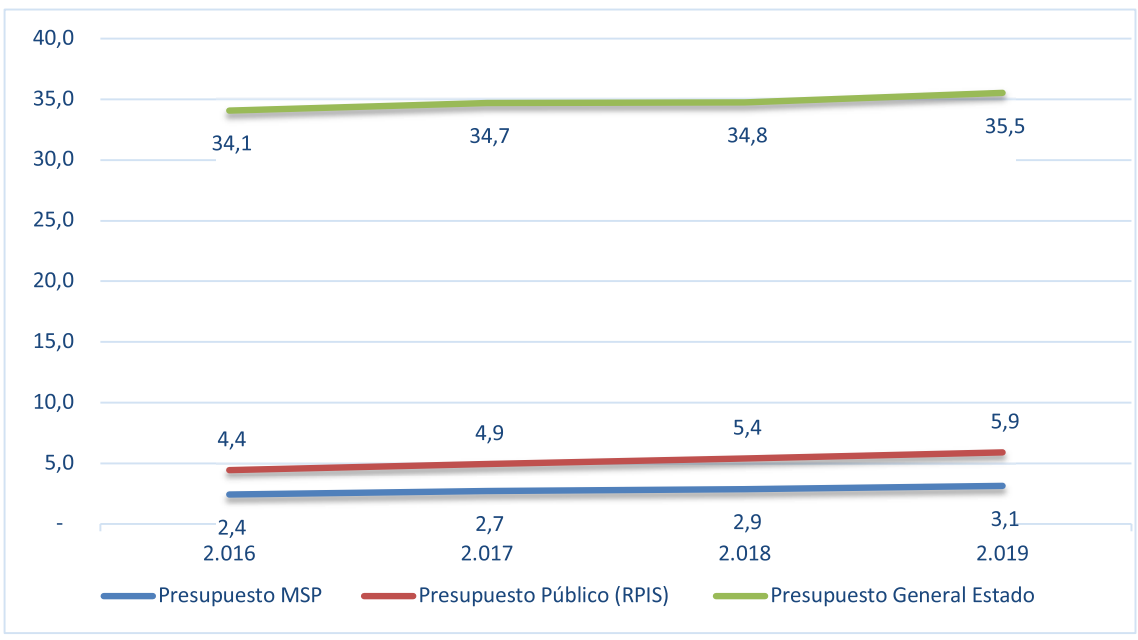

Fuente: E-sigef, Banco Central del Ecuador

En este contexto es importante, en contraste con la desaceleración en la tasa de participación del PGE en relación al PIB, observar que, los presupuestos de los últimos años de los subsistemas públicos de salud han logrado crecer particularmente anclados en los ingresos provenientes externos al fisco. Esto se puede constatar al comparar las proporciones respecto al PIB alcanzadas por los otros subsistemas públicos, que no dependen solamente de recursos fiscales. El financiamiento gubernamental no creció al ritmo del crecimiento de las otras fuentes financiadoras, como las contribuciones personales y patronales, la diferencia entre las proporciones de los presupuestos institucionales respecto al PIB se acentúa pasando de 2,01\% en 2016 a 2,44\% en 2019, lo que significa una mayor participación de los hogares en el financiamiento global de la salud en Ecuador. 
Tabla 2. Porcentaje de presupuesto y Diferencia porcentual presupuestaria de la RPIS y MSP en relación al PIB

(2016-2019)

\begin{tabular}{|c|c|c|c|}
\hline Año & \% PrsRPIS/PIB & \% MSP/PIB & Dif (\%RPIS/PIB - \%MSP/PIB) \\
\hline 2016 & $4,44 \%$ & $2,43 \%$ & $2,01 \%$ \\
\hline 2017 & $4,74 \%$ & $2,61 \%$ & $2,12 \%$ \\
\hline 2018 & $4,94 \%$ & $2,63 \%$ & $2,31 \%$ \\
\hline 2019 & $5,22 \%$ & $2,78 \%$ & $2,44 \%$ \\
\hline
\end{tabular}

Fuente: BCE valores corrientes, PIB 2016 y 2017 es semidefinitivo y provisional Cuentas Nacionales Anuales; PIB 2018 y 2019 Previsiones Macroeconómicas 2018. Presupuesto MSP, MIDENA y Ministerio del Interior se toman del MEF-Esigef. Presupuesto del IESS se toma de la Resoluciones del Consejo Superior

\section{c. Per cápita de Salud en Ecuador}

El promedio regional invertido por persona en salud asciende en 2016 a USD 606,88. Ecuador se encuentra por debajo del promedio con USD 505. Si asumimos que el financiamiento público es de alrededor del $51 \%$, el per cápita anual público invertido en salud ascendería tan solo a USD 258 en 2016, cifra que solventa uno de los modelos de atención más completos y universales de la región. En 2019 el rubro de gasto público en salud se proyecta en USD 342 por cada habitante. 


\section{Gráfico 8. Gasto en Salud Per Cápita en Latinoamérica (USD 2016)}

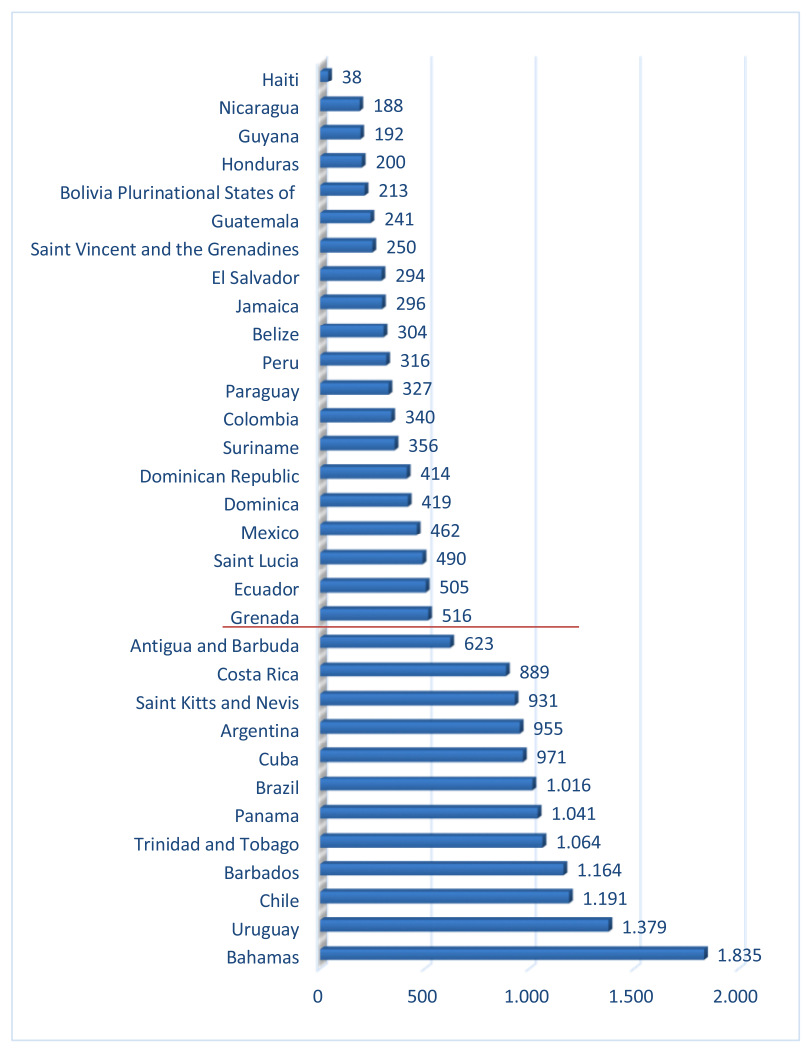

Fuente: (OMS, n.d.) consultado 05 marzo 2019

Con lo anterior, y entendiendo a con una inversión baja por habitante, el la salud como un bien público ${ }^{6}$, corres- ${ }^{-}$sistema se orienta a mejoras sustanciales ponde al gobierno realizar un ejercicio de eficiencia en su producción y ahorros regulatorio sistémico. Es decir, evaluar si en su desempeño.

\footnotetext{
${ }^{6}$ Un Bien Público es aquel que tiene dos propiedades básicas: no cuesta nada que otra persona más disfrute de sus ventajas es decir tiene un coste marginal nulo y es difícil o imposible impedir que se disfrute del bien público. El mercado o bien no suministra los bienes públicos puros, o bien no los suministra en una cantidad suficiente (Stiglitz, 2003).
} 


\section{d. Estructura financiera, oferta y co- berturas}

El modelo de salud ecuatoriano ha organizado su financiamiento a través de un Sistema Nacional de Salud (National Health System) ${ }^{7}$, cuyo financiamiento se apalanca principalmente en los impuestos de la ciudadanía y regalías estatales, y es ejecutado a través de transferencias fiscales a los entes administradores de recursos y, estos a su vez, a los prestadores de servicios de salud. Por otro lado, el país también ha organizado su financiamiento a través de un sistema de seguridad social público compulsorio (National Insurance System) ${ }^{8}$, que funciona a través de contribuciones patronales y personales obligatorias en los subsistemas del IESS, ISSFA, ISSPOL. Dichas contribuciones se apalancan fundamentalmente en recursos generados por la ciudadanía, puesto que económicamente los hogares son la cantera de las empresas, por lo cual contribuyen directamente con sus aportes personales, pero también a través de los aportes que las empresas realizan como patronos. Por lo anterior, en el corto y mediano plazo, y sin conocimiento claro de su aporte real, existirán fuertes tensiones por parte de la población, para exigir un mayor alcance efectivo en la oferta y un mejor rendimiento de las inversiones prestacionales, lo cual tensionará el presupuesto gubernamental que trata al sector salud como un "gasto" más que tiene que afrontar, al igual que los demás sectores, sin entender el real aporte que esta se encuentra realizando ya a la economía en la restitución del bienestar a los hogares.

Con lo señalado y a manera de concretar las figuras, el financiamiento del Sistema Nacional de Salud (SNS) en Ecuador se articula a través de siete subsistemas: i. Gobierno-MEF-MSP, ii. IESS-SGSFI, iii. IESS-SSC, iv. MIDENA-ISSFA, v. MDI-ISSPOL, vi. MDTOP-ANT-SPPAT y vii. Privado Sin y Con Fines de Lucro, quienes en primera instancia son los encargados de capturar y compilar los recursos provenientes de tres fuentes: Gobierno, Hogares y Organismos Internacionales, en una variedad de modalidades de recursos que provienen de fuentes no excluyentes ni exclusivas, de carácter público, privado o mixto.

\footnotetext{
${ }^{7}$ National Health System, más conocido como modelo Beveridge es uno de los dos modelos icónicos o referentes de cómo se organiza el financiamiento en salud en un país. Este modelo basa la titularidad del derecho (al servicio de salud) en la ciudadanía, y se fondea a partir de los impuestos de ciudadanos y recursos del estado. Inglaterra es el país representativo de este tipo de arreglos financieros para salud.

${ }^{8}$ National Insurance System, conocido como modelo Bismarckiano es el otro modelo referente y se basa en la titularidad del derecho basado en la afiliación laboral, suponiendo que toda la población se incorporará al mercado laboral, desde donde podrán realizar contribuciones para la seguridad social.
} 
Los subsistemas mencionados brindan una cobertura efectiva a más realizan también la administración de los recursos financieros acopiados. Con estos adquieren atenciones de salud para las poblaciones que les competen, sea bajo la titularidad de afiliado o de ciudadano beneficiario. La adquisición de atenciones de salud se realiza a través de prestadores propios o prestadores externos, siendo los primeros establecimientos o facilidades cuya manutención global —especialmente del talento humano- es asumida bajo la figura de patrono. Los segundos son establecimientos o profesionales a quienes se les reconoce un valor por la prestación de salud realizada.

Los subsistemas públicos organizados en la denominada Red Pública Integral de Salud (RPIS) ${ }^{9}$ se estima que

del 90 \% de la población ecuatoriana, y en conjunto con el sector privado alcanzarían a proporcionar la cobertura universal, aunque todavía no el acceso universal. Es importante mencionar que la cobertura de salud privada mostrada en las encuestas de hogares oscila en torno al $3 \%$ de la población cuando se consideran los factores de expansión. Esto sucede ya que este tipo de encuestas no logran capturar una muestra representativa de los hogares de los quintiles más ricos. Ello ocasiona que el dato que se obtenga en cuanto a cobertura tenga un posible sesgo de selección y tienda a estar subestimado. Por este motivo, y pese a ser un dato de 2010, esta cobertura se toma del último Censo de Población y Vivienda realizado en el país.

\footnotetext{
${ }^{9}$ La RPIS nace de la Constitución de la República, a la par de refrendarse con un Convenio firmado por todos los subsistemas en 2012. Tiene como objeto establecer mecanismos de articulación entre instituciones públicas prestadoras y aseguradoras de salud para complementar la cobertura de servicios nacional.
} 


\section{Gráfico 9. Cobertura efectiva de atención de salud por subsistema}

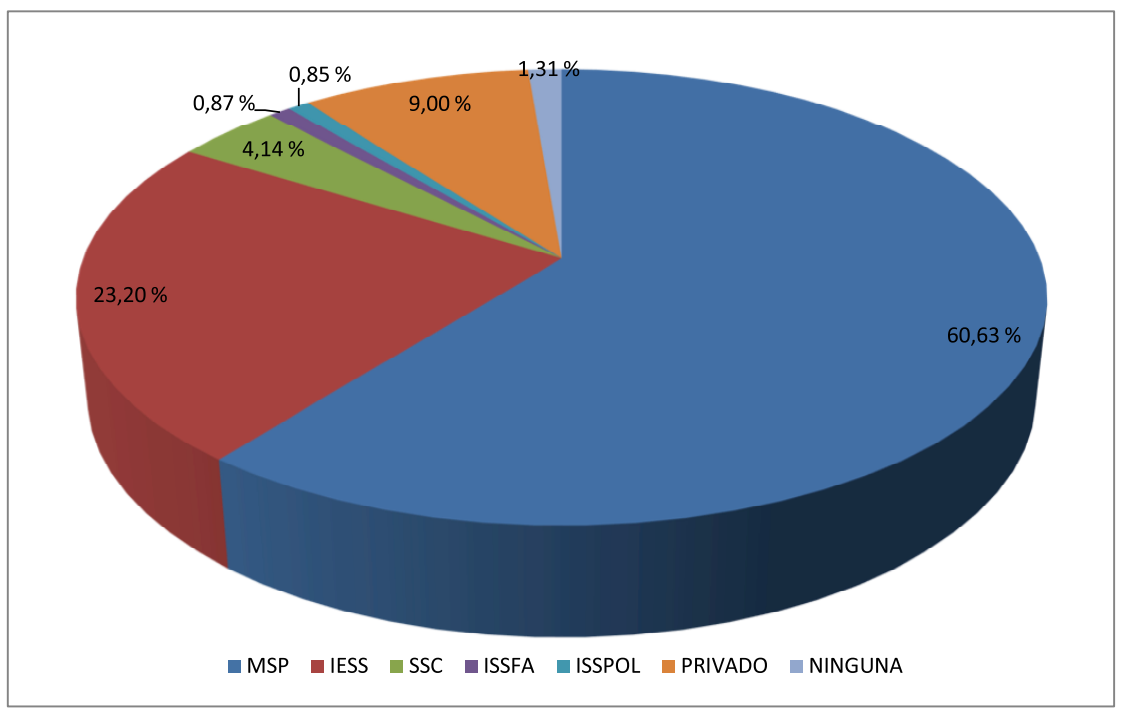

Fuente: E-sigef, IESS,INEC

Los subsistemas públicos brindan una atención directa a sus derechohabientes y, a partir de la conformación de la RPIS en marzo de 2012, también intercambian atenciones de salud entre sí a pacientes derivados de la Red, sea por falta de capacidad física o técnica. En los últimos años la RPIS también derivó pacientes a la Red Complementaria (RC) o subsistema privado. Se estima que para el año 2018, solamente el MSP compró servicios de hospitalización, emergencia, diálisis-hemodiálisis y ambulatorios, por un monto superior a los USD 406 millones (MSP-DNA, 2018).
Respecto al financiamiento de las coberturas brindadas por los subsistemas públicos, en el Ecuador, se estima que el Gobierno, entre presupuestos del MSP y entidades adscritas, Ministerio de Defensa Nacional (MIDENA), Hospital de Especialidades de Fuerzas Armadas, Ministerio del Interior (MDI), Dirección Nacional de Salud de la Policía Nacional y las contribuciones a la seguridad social como patrono, se encontraría aportando para 2019 alrededor de USD 4 mil millones en salud. En este rubro se estarían considerando también los recursos que están ingresando al país por la vía de los Organismos Internacionales. 
Los hogares por su parte realizan compras de servicios y productos de salud estimados al menos en USD 3,7 mil millones, pago de seguros de enfermedad y accidente y medicina prepagada USD 500 millones, aportes a la seguridad social voluntaria y como cotizantes en los seguros sociales públicos de fuerzas armadas y policía así como aportes patronales de las familias USD 1,5 mil millones, pago de impuesto a créditos del sistema bancario destinados a cáncer USD 100 millones, juegos de lotería nacional y otros destinados a salud por alrededor de USD 75 millones y una prima anual para pago de accidentes de tránsito por alrededor de USD 30 millones, rubros que lindarían en alrededor de USD 6 mil millones para 2019.

\section{e. Establecimientos de Salud}

Los recursos mencionados solventan la cartera de servicios de las instituciones que conforman la RPIS, en la cual se identifica que el MSP como el prestador más grande de Ecuador, brinda servicios generales y de salud pública a través de 2.096 establecimientos de salud. El IESS cuenta con 108 establecimientos, el ISSPOL e ISSFA brindan servicios especializados y disponen de 56 y 103 establecimientos, respectivamente. Finalmente, el Seguro Social Campesino del IESS dispone de 658 establecimientos ambulatorios (Instituto Ecuatoriano de Estadísticas y Censos, n.d.).

Tabla 3. Número de establecimientos de Salud del MSP por Tipología

\begin{tabular}{|l|c|}
\hline \multicolumn{1}{|c|}{ TIPOLOGÍA } & CANTIDAD \\
\hline HOSPITALES DE ESPECIALIDADES & 3 \\
\hline CENTROS AMBULATORIOS ESPECIALIZADOS & 24 \\
\hline HOSPITALES ESPECIALIZADOS & 12 \\
\hline HOSPITALES GENERALES & 32 \\
\hline HOSPITALES BÁSICOS & 86 \\
\hline HOSPITALES MÓVILES & 2 \\
\hline CENTROS DE SALUD & 1.939 \\
\hline TOTAL ESTABLECIMIENTOS DE SALUD & 2.096 \\
\hline
\end{tabular}

Fuente: Número de establecimientos, Geosalud-Ministerio de Salud Pública 
Adicionalmente, el propio sistema presenta presiones que lo obligan a buscar nuevas fuentes de financiamiento para preservar su sostenibilidad tanto económica como técnica a efectos de responder a nuevos retos como el envejecimiento de la población y el aumento de la esperanza de vida (74 años). Las generaciones más jóvenes están menos interesadas en tener hijos, eso lo demuestra la reducción de las tasas de crecimiento poblacional. Entre los censos de 1990 a 2001 fue de 2,10\%, mientras que entre el censo del 2001 y 2010 el crecimiento fue de $1,95 \%$; la tasa de fecundidad también ha decrecido. Otra presión es el perfil epidemiológico de Ecuador. Así mismo se evidencia una transición hacia enfermedades no transmisibles y crónicas que involucran una mayor cantidad de recursos económicos para su tratamiento. Por último, la política pública de Ecuador ha trabajado por tener ciudadanos empoderados y conocedores de sus derechos en torno a la salud, esto ejerce una presión social hacia la demanda de estos servicios, a lo cual se suma las mismas presiones de una oferta producto de una organización fragmentada y segmentada de los servicios de salud. Todo lo mencionado afecta la capacidad para brindar atención sanitaria continua, oportuna e integral, generando costos cada vez más elevados y una ineficiente asignación de los recursos en el sistema.
La situación de Ecuador no es aislada. En la región la sostenibilidad en la salud comienza a ser un tema generalizado por razones como la eficiencia de los recursos, los costos de oportunidad y la sostenibilidad de los logros ya alcanzados. La sostenibilidad del financiamiento depende básicamente de dos componentes. El primero es aumentar el espacio fiscal para la salud. El espacio fiscal hace referencia a la capacidad del Gobierno a asignar recursos presupuestarios adicionales para la RPIS sin afectar la situación financiera del sector público ni desplazar otros gastos que son socialmente necesarios. El segundo componente es la generación de mecanismos estables de financiamiento para la salud. La idea es cambiar una norma social en el Ecuador y hacer que todos los ecuatorianos se sientan corresponsables de mantener un sistema sanitario de calidad. La RPIS debe brindar servicios de salud integrales de calidad cuando lo necesiten, como un solo cuerpo. Para ello, el sistema debe asegurar el origen y la fuente de los recursos económicos para que esté en capacidad de cubrir a toda la población y se elimine la capacidad de pago como barrera de acceso a los servicios de salud.

Los problemas financieros ciertamente se atacan desde la generación de mayores recursos, consolidación de fuentes de financiamiento, pero también 
y principalmente a través de mejoras en la eficiencia del gasto y logro de ahorros. En este punto es importante anotar que según un informe de la Organización Mundial de la Salud en 2010, las ineficiencias causan entre un 20 a $40 \%$ de desperdicios en el gasto total en salud (OMS, 2010; OPS/OMS, 2014).

Al establecer acciones coordinadas entre los diferentes subsistemas, en el primer nivel de atención, se pueden generar ahorros disminuyendo admisiones hospitalarias evitables, readmisiones, atenciones de urgencia y emergencia. Adicionalmente existirían menos costos administrativos por la reducción de procesos de contratación, se incurriría en economías de escala ya que un mayor volumen de compra permite tener un mejor precio y mayor poder de negociación, se generarían ahorros por la disminución de desperdicios de medicamentos caducados, ahorros en el almacenamiento y distribución de medicamentos e insumos médicos ya que al realizar compras centralizadas solo se requiere una adecuada administración de operaciones. Por ello es central crear una instancia coordinadora nacional de acompañamiento en la gestión técnica a la vez que establece el control técnico necesario para evitar duplicaciones y desperdicios en la atención sanitaria, producto de que varias entidades se encuentren a cargo del financiamiento de las atenciones. Con ello se evita también el tratamiento diferenciado a los beneficiarios de los servicios de salud que actualmente se está experimentando, así como el uso inadecuado de los servicios, especialmente de los de mayor complejidad que son los que generan gastos más elevados, conduciendo de manera técnica al pago por resultados, mecanismo efectivo para corregir ineficiencias, superar la fragmentación del sistema y evitar espacios de corrupción.

\section{f. Composición del presupuesto del MSP}

El subsistema de salud financiado por el gobierno a través del MSP, ha tenido una evolución creciente. Sin embargo, debido a las restricciones fiscales en los últimos años tiende a desacelerar fuertemente su crecimiento 


\section{Gráfico 10. Presupuesto devengado MSP/ PIB (Miles de Millones USD)}

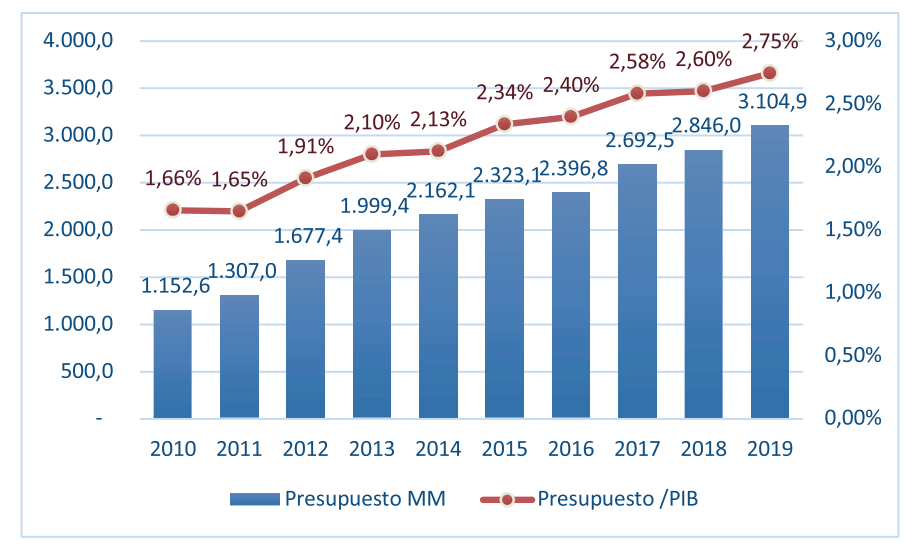

Fuente: MEF-E-sigef presupuesto devengado 2010-2018, asignado 2019

En este punto es necesario, a efectos de lograr precisar el gasto efectivo en atención de la salud, realizar un ejercicio de desagregación del presupuesto desde una perspectiva funcional. Con ello se puede encontrar que el presupuesto del Ministerio de Salud Pública no responde únicamente a asegurar el funcionamiento del subsistema de atención más gran- de del país, sino que a la par incorpora el desarrollo de la rectoría sectorial, esto es la orientación, conducción y normatización de la salud (8\%), y además financia el desarrollo de los Programas de Salud Pública (4\%) fundamentales para disminuir los riesgos y morigerar los determinantes sociales. 


\section{Grafico 11. MSP composición del presupuesto por funciones}

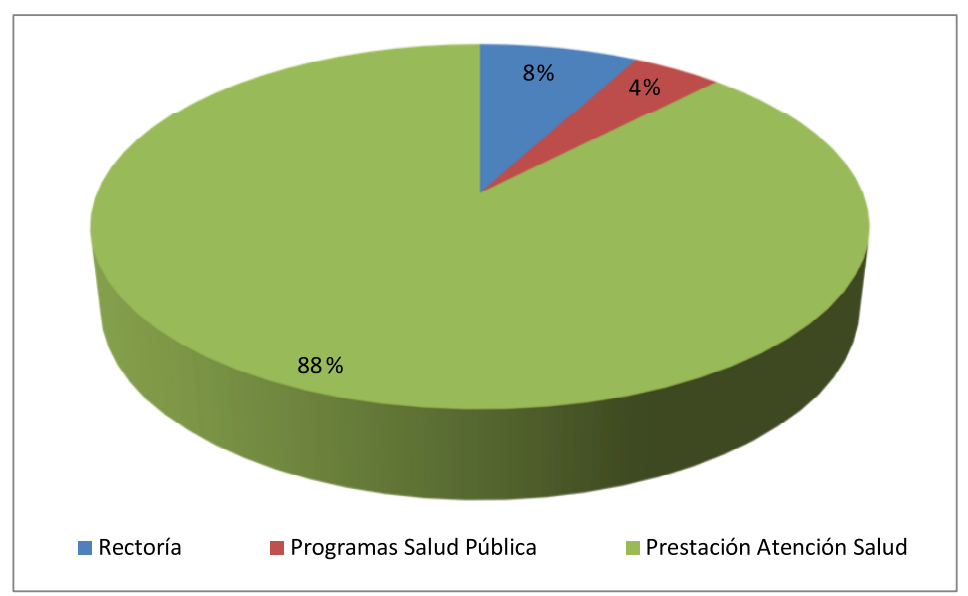

Fuente: E-sigef. Autor: Dirección de Economía de la Salud-MSP

Lo mencionado se traduce al hecho de que una afectación al financiamiento público del MSP —a diferencia de los otros subsistemas - influye no solo a la provisión individual de salud, sino a la provisión colectiva de programas que aseguran el bienestar de toda la población.

El meollo del asunto pasa por el hecho de que, en países con una frágil institucionalidad y debilidad técnica y tecnológica, el ejercicio regulatorio se sustenta en mayor grado en la capacidad de financiar el sistema, por lo que al dis- minuir el presupuesto dicha capacidad podría disminuir también.

En lo que respecta a la composición del presupuesto del MSP, por grupo de gasto, se puede observar en el detalle del devengado 2018, que el mayor porcentaje se destina a personal (60\%), seguido de insumos (8,5\%) y medicamentos (5,3\%), lo cual responde a la lógica que en el sector salud, el talento humano es el recurso prioritario y necesario por excelencia y posiblemente el único imprescindible. 


\section{Gráfico 12. MSP Presupuesto Devengado 2018 por Grupo de Gasto}

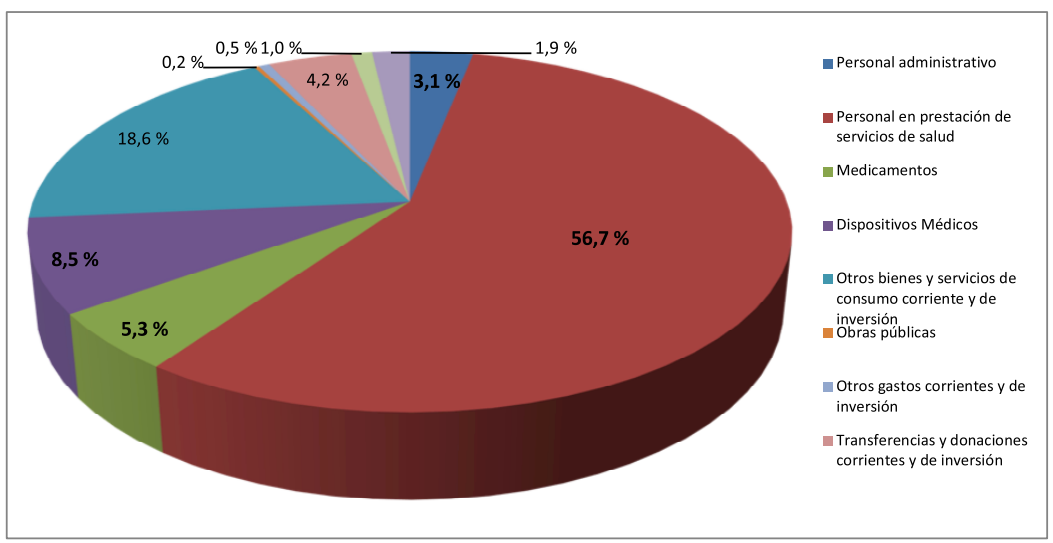

Fuente: E-sigef

\section{g. Talento Humano en Salud}

El Gobierno, a través del MSP destinó para 2018 alrededor de USD 1700 millones para Talento Humano, lo cual representó alrededor del $60 \%$ del presupuesto devengado de ese año. Un análisis tendencial señala que, durante la última década, el promedio de recursos presupuestado destinando a talento humano ha fluctuado entre el $56 \%$ y el $68 \%$ del total.

Bajo la premisa de que para que un Sistema Nacional de Salud funcione, el talento humano en salud debe ser receptivo, equitativo y eficiente; en función de las circunstancias y recursos existentes es importante entender que, en términos económicos, un SNS está sujeto a una restricción presupuestaria que debe permitir que el número de personal asistencial en los establecimientos de salud sea suficiente y esté disponible (OTHS Colombia, 2015), sin que esto ponga en riesgo su sostenibilidad financiera.

El crecimiento y desarrollo del ámbito sanitario depende básicamente de una fuerza de trabajo apropiada, personal calificado, con competencias adecuadas, ya que el esfuerzo que imprimen los trabajadores impactará directamente en los resultados por la política pública. Dussault afirmaba que los costos económicos y humanos, de tener pobres recursos humanos en salud, son particularmente altos en el sector salud a consecuencia de que la eficiencia, eficacia y desempeño depende básicamente de quien provee el servicio, en este caso el 
personal asistencial directamente (Dussault \& Dubois, 2003) increasing attention has been paid to the development of health policies. But side by side with the presumed benefits of policy, many analysts share the opinion that a major drawback of health policies is their failure to make room for issues of human resources. Current approaches in human resources suggest a number of weaknesses: a reactive, ad hoc attitude towards problems of human resources; dispersal of accountability within human resources management (HRM.

EI MSP como ente rector asume la tarea de promover los servicios de salud mediante la repotenciación y creación de nuevos establecimientos de salud y con ello la incorporación de nuevo personal cualificado y preparado, en número suficiente para brindar una atención oportuna de calidad, tal y como lo dispone el Modelo de Atención Integral en Salud (MAIS) (Ministerio de Salud Pública. Subsecretaría Nacional de Gobernanza de la Salud Pública., 2013). El MAIS al institucionalizarse en 2012 enfatiza en generar un cambio del enfoque de la salud pública hacia la atención primaria en salud y con ello un cambio desde lo curativo hacia lo preventivo, lo cual impacta directamente en la forma en que las personas son atendidas en los establecimientos de salud, ya que se estableció una nueva cartera de servicios en función a la también nueva tipología de los establecimientos de salud, logrando una adaptación de los perfiles asistenciales necesarios para cubrir las nuevas vacantes generadas, dando paso a una reconfiguración total del servicio.

En este punto es importante desde el punto de vista económico entender la organización sanitaria como una organización productiva. Es decir, los servicios están integrados por centros de producción que generan procesos productivos con criterios de análisis cuantificables y medibles, que se clasifican en:

1. Servicios Finales y de apoyo asistenciales: son centros que mantienen una relación directa médico / paciente.

2. Servicios de Apoyo o Complementarios: son centros que apoyan al cumplimiento de los objetivos de los establecimientos asistenciales.

- Servicios de apoyo Complementario (Laboratorio, imagen, entre otros).

- Servicios de apoyo Logístico (Mantenimiento, bodega, farmacia, entre otros).

- Servicios de apoyo administrativo (Administración).

En 2019 el número de personas empleadas a nivel de hospitales generales, de especialidad y especializados 
es de alrededor de 31 mil servidores públicos entre Servicio Asistencial, Apoyo Asistencial, Apoyo Complementario, Apoyo Administrativo, Apoyo Logístico y Jerárquico Superior.

Un rápido análisis institucional señala que el Hospital de Especialidades Eugenio Espejo -el más grande en tamaño y complejidad del subsistema MSP — es el que tiene la mayor cantidad de personas empleadas (2.036), seguido por el Hospital de Especialidades Abel Gilbert Pontón (1.697) y Pediátrico Francisco Icaza Bustamante (1.352).
Al analizar la composición del talento humano en cada una de las tipologías de hospital, se puede observar que esta es similar. Así el tema asistencial se lleva la mayor parte del talento humano (médicos, odontólogos, obstetras, psicólogos y terapeutas) y oscila entre el $20,5 \%-26,9 \%$. Por otro lado, el personal dedicado a servicios de apoyo asistencial (enfermería) varía entre el 32,7\% - 37,5\%. El apoyo administrativo oscila entre el $21 \%-28 \%$.

\section{Gráfico 13. Composición del talento humano por tipología de hospital del MSP}

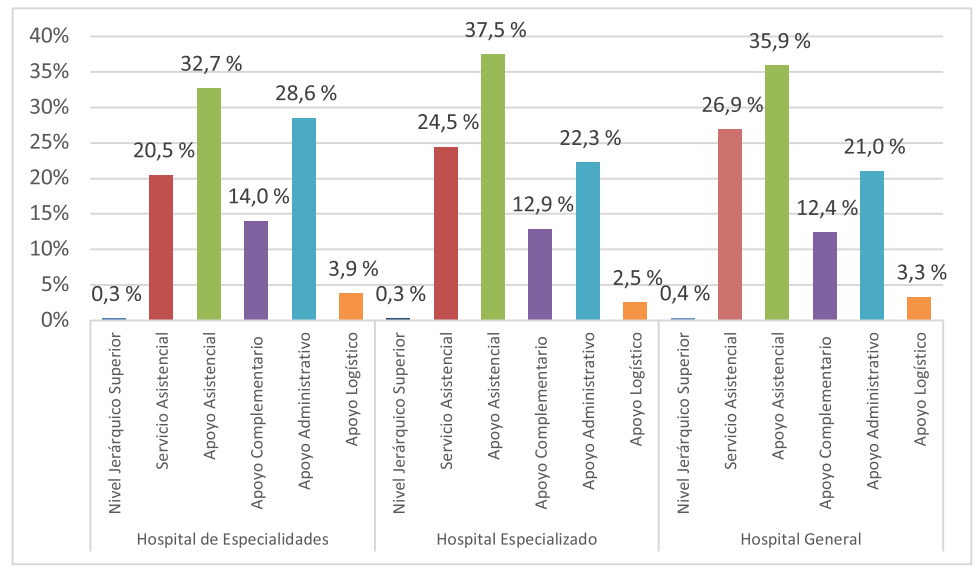

Fuente: Distributivo del Talento Humano de los Hospitales. Elaboración: Autores

Por otro lado, si se pone en perspectiva la distribución geográfica de hospitales públicos de Especialidades,
Especializados y Generales, se refleja un resultado bastante intuitivo relacionado con que aquellas provincias con ma- 
yor densidad poblacional más recursos humanos en salud. Guayas (3.116.111 hab.), Pichincha (4.267.893 hab.) y Manabí (1.537.090 hab.) concentran más personal asistencial especializado que el resto de las provincias. El resto de las provincias evidencian una distribución bastante homogénea. Según datos de la Encuesta de Recursos y Actividades en Salud del INEC, entre 2000 y 2016 la tasa de médicos mantiene una tendencia creciente y se incrementa en aproximadamente 12 puntos porcentuales, es decir que para el 2016 hay 21 médicos por cada 10.000. La siguiente ilustración muestra la existencia de profesionales de la salud a nivel nacional, sin embargo, persiste una brecha de profesionales requeridos en las unidades operativas a nivel nacional.

\section{Distribución Geográfica del TT HH y Sueldos y Salarios. Mapa de Calor}

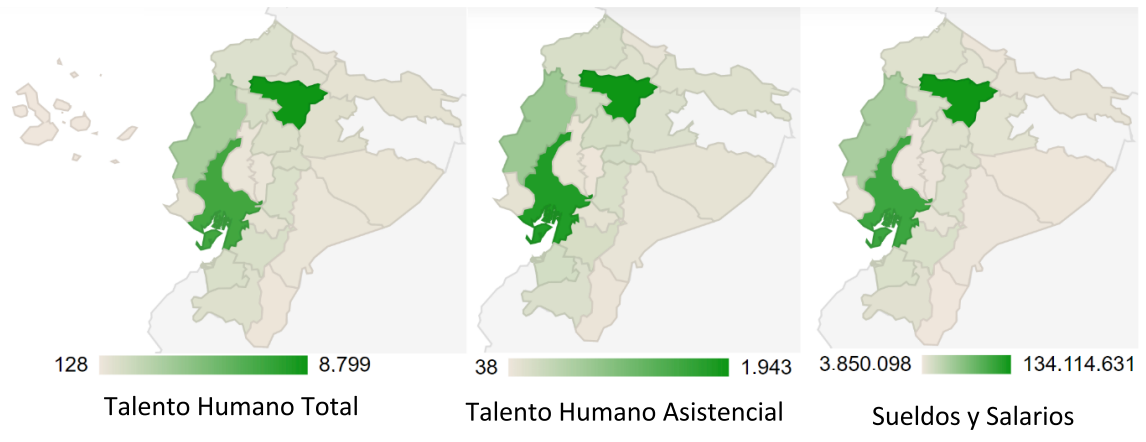

Fuente: Distributivo del Talento Humano de los Hospitales

En los últimos años, el sector salud ha sufrido varias modificaciones en lo que respecta a la determinación de sueldos y salarios, el hecho más significativo en esta materia fue el acercamiento —que solamente se logró en los primeros años de la década - entre los diferentes subsistemas financiados desde el Gobierno, con base en un esquema financiado por impuestos, y el subsistema de seguridad social financiado por contribuciones; en los dos casos ambas fuentes provenientes en mayor medida de los hogares y empleadores. Sin embargo, anclado en una falsa percepción de disponibilidad de recursos, pronto los salarios de la seguridad social tendieron a diferenciarse, en el afán de captar una mayor cantidad de talento humano para ese 
subsistema. A la fecha, a diferencia de los hospitales del IESS, que cuentan con un mayor número de recursos y mejores salarios, en el subsistema del MSP encontramos el esquema señalado en la tabla 4.

\section{Tabla 4. Remuneraciones Promedio del Talento Humano en Hospitales del MSP}

\begin{tabular}{l|c|} 
Personal Asistencial & RMU promedio USD \\
\hline Gerente de Hospital & $4.235,35$ \\
\hline Gerente de Hospital Especializado & $6.191,00$ \\
\hline Médico General & $2.301,70$ \\
\hline Médico Especialista & $2.666,58$ \\
\hline Médico cirujano & $2.337,50$ \\
\hline Médico Anestesiólogo & $2.641,00$ \\
\hline Enfermera /o & $1.266,94$ \\
\hline Auxiliar de enfermería & 797,45 \\
\hline Laboratorista Clínico & $1.352,59$ \\
\hline Auxiliar de Laboratorio & 779,14 \\
\hline Odontólogo Especialista & $1.990,52$ \\
\hline Odontólogo & $1.748,54$ \\
\hline Auxiliar de Odontología & 820,29 \\
\hline Obstetriz & $1.580,41$ \\
\hline Paramédico & 937,08 \\
\hline Psicólogo Clínico & $1.469,05$ \\
\hline Nutricionista & $1.262,98$ \\
\hline Químico & $1.392,93$ \\
\hline Tecnólogo médico & $1.171,69$ \\
\hline
\end{tabular}

Fuente: Distributivo del Talento Humano de los Hospitales. s.

Si tomamos en cuenta que los salarios son un elemento primordial en la atención de la salud, así como también la satisfacción personal en torno a la realización profesional, productividad e incentivos que estos puedan generar en los profesionales de la salud (RISSALUD,
2017), es claro que contar con un nivel de salarios competitivo en el sector público podría garantizar que los médicos presten sus servicios públicos y con ello se garantiza la seguridad sanitaria nacional. De continuar la tendencia, no será el caso ecuatoriano. 
En cuanto a la masa salarial, el Ministerio de Salud Pública destina un total de USD 623,2 millones de dólares anuales para mantener en funcionamiento estos tres tipos de hospitales. El gasto administrativo como porcentaje del gasto total oscila entre $12,72 \%$ y el 18,49\% incrementándose de acuerdo a la complejidad del hospital. De igual forma, esto guarda concordancia con el número de personal asistencial que tiene cada una de dichas casas de salud.

Tabla 5. Masa Salarial Total en Hospitales del MSP por Tipología

\begin{tabular}{|c|c|c|}
\hline Hospitales de Especialidades & $77.869 .843,46$ & $12,50 \%$ \\
\hline Nivel Jerárquico Superior & $621.082,60$ & $0,80 \%$ \\
\hline Servicio Asistencial & $29.294 .835,87$ & $37,62 \%$ \\
\hline Apoyo Asistencial & $21.627 .231,17$ & $27,77 \%$ \\
\hline Apoyo Complementario & $9.919 .685,04$ & $12,74 \%$ \\
\hline Apoyo Administrativo & $14.401 .559,80$ & $18,49 \%$ \\
\hline Apoyo Logístico & $2.005 .448,98$ & $2,58 \%$ \\
\hline Hospitales Especializados & $142.182 .900,17$ & $22,82 \%$ \\
\hline Nivel Jerárquico Superior & $1.027 .346,34$ & $0,72 \%$ \\
\hline Servicio Asistencial & $59.425 .250,60$ & $41,79 \%$ \\
\hline Apoyo Asistencial & $44.531 .399,85$ & $31,32 \%$ \\
\hline Apoyo Complementario & $15.820 .547,46$ & $11,13 \%$ \\
\hline Apoyo Administrativo & $19.202 .349,49$ & $13,51 \%$ \\
\hline Apoyo Logístico & $2.176 .006,43$ & $1,53 \%$ \\
\hline Hospitales Generales & $403.140 .490,70$ & $64,69 \%$ \\
\hline Nivel Jerárquico Superior & $3.338 .329,73$ & $0,83 \%$ \\
\hline Servicio Asistencial & $177.579 .837,49$ & $44,05 \%$ \\
\hline Apoyo Asistencial & $121.354 .124,02$ & $30,10 \%$ \\
\hline Apoyo Complementario & $41.351 .957,36$ & $10,26 \%$ \\
\hline Apoyo Administrativo & $51.269 .866,01$ & $12,72 \%$ \\
\hline Apoyo Logístico & $8.246 .376,09$ & $2,05 \%$ \\
\hline Total & $623.193 .234,32$ & $100 \%$ \\
\hline
\end{tabular}

Fuente: Distributivo del Talento Humano de los Hospitales. Elaboración: Autores 


\section{MEDICAMENTOS Y DisPOSITIVOS MÉdiCOS}

Históricamente, por su naturaleza, el servicio de salud conllevó una mayor carga en el concurso del talento humano. Tal naturaleza se relaciona con la posibilidad de brindar una prestación, básicamente a través del conocimiento que los profesionales de la salud poseen, aun en condiciones de limitaciones 0 precariedad. No obstante, la cuarta revolución industrial por la cual estamos atravesando, propicia por primera vez la posibilidad de revertir esa relación y balancear el presupuesto hacia la compra de dispositivos médicos o fármacos innovativos, que constituyen una ruptura tecnológica con el pasado, antes que incrementar la inversión en talento humano.

El cambio tecnológico actual, comparable a la introducción de la máquina de vapor en 1772, o de la bombilla eléctrica en 1880, representa también el crecimiento de los recursos invertidos en nuevas tecnologías ${ }^{10}$. Así, solamente el Gobierno a través del MSP destinó para 2018 alrededor de USD 150 millones para medicamentos y USD $241 \mathrm{mi}$ llones para dispositivos médicos, lo cual representó alrededor del 5,3\% y 8,5\% del presupuesto devengado de ese año, respectivamente, y sumados alrededor del 14\%. Rubros inéditos en décadas previas.

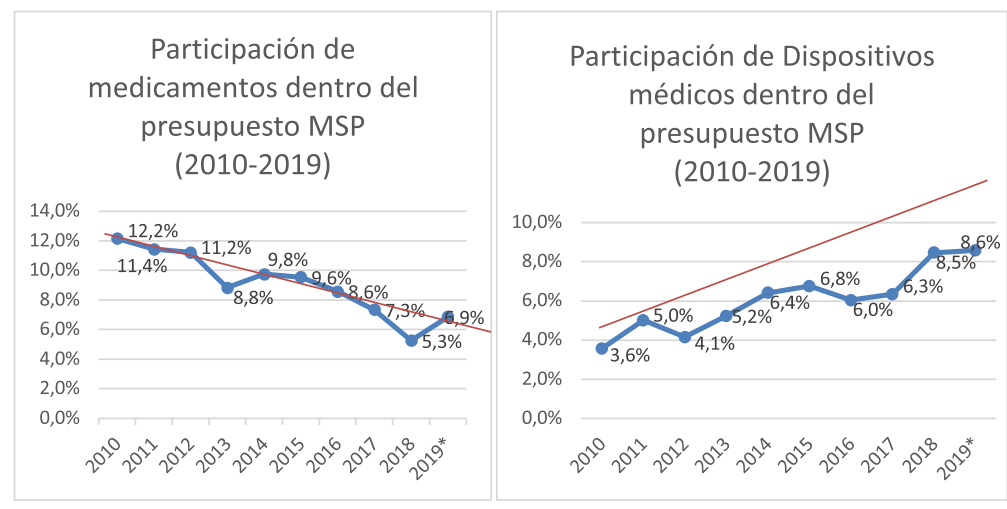

Fuente: E-sigef MEF (Ministerio de Finanzas, n.d.)

\footnotetext{
${ }^{10}$ Entendemos como tecnología sanitaria a la aplicación de conocimientos teóricos y prácticos estructurados en forma de dispositivos, medicamentos, vacunas, procedimientos y sistemas elaborados para resolver problemas sanitarios y mejorar la calidad de vida. Resolución WHA60.29 Asamblea Mundial de la Salud, mayo 2007.
} 
Es importante matizar que, aunque existe crecimiento en la participación de tecnologías sanitarias como medicamentos y de dispositivos médicos, también existe una disputa de recursos dentro de un presupuesto limitado, sin embargo, es claro que la primacía del ítem presupuestario de talento humano, cede espacio a las necesidades de contar para su desempeño con capacidad diagnóstica o farmacológica adicional.

\section{Costo de oportunidad de la compra de medicamentos de alto impacto financiero en el Ecuador}

En el contexto mencionado, no podemos dejar de señalar un aspecto que influye en el manejo de tratamiento, y es el considerar que la RPIS utiliza los medicamentos que se encuentran en el Cuadro Nacional de Medicamentos Básicos (CNMB), puesto que estos son los que se financian desde el Gobierno ecuatoriano. Esto es más claro cuando se produce una tensión para tratar enfermedades raras, para las cuales no se han desarrollado o incluido medicamentos en el CNMB, donde se vuelve muy costosa la innovación relacionada al tratamiento de la misma, y donde desde diversos espacios se requieren nuevos medicamentos que introducen presiones financieras por el lado de la demanda - hogares o médicos prescriptorespara su inclusión (financiamiento), que finalmente pesa sobre las decisiones de adquisición de los administradores de recursos de atención de salud.

Es claro también que el incremento anual en el presupuesto no necesariamente se traduce en el crecimiento proporcional de todos sus componentes. De tal manera que, dependiendo de la composición del gasto en salud, el incremento de uno de estos componentes puede traducirse en la disminución de los recursos destinados para los demás. Asimismo, el incremento de rubros en un subsistema genera presiones en los otros subsistemas públicos y/o privados, puesto que el agotamiento de ciertas partidas como las de medicamentos o dispositivos médicos, hace que la población demande su atención, aun en aquellos en los cuales no posee titularidad. Específicamente en el caso de medicamentos, la inclusión de un medicamento cuyo precio sea superior a la media del costo de los medicamentos esenciales, reducirá el presupuesto en medicamentos restante. Esto podría ocasionar que no se puedan comprar otros medicamentos esenciales de alta calidad, seguros, eficaces y asequibles. Considerando que adquirir este tipo de medicamentos es una de las bases para el correcto funcionamiento de los sistemas de salud, el no contar con estos medicamentos podría afectar los resultados de salud, que buscan lograr una cobertura universal. $\bigcirc$ 
en su lugar, se podrían realizar recortes para otros programas de salud pública".

Los cambios demográficos, en particular el envejecimiento de la población con aumentos en la prevalencia de enfermedades crónicas, exacerba el problema del acceso a la salud. El conflicto entre, por un lado adquirir medicamentos de alto impacto financiero y, por otro lado, los presupuestos limitados de medicamentos es una fuente constante de tensión política. Aunque negar el tratamiento es una cuestión bastante polémica, es necesario también analizar esta situación desde la perspectiva de salud pública y realizar evaluaciones económicas, tales que permitan utilizar evidencia robusta para recomendar o no su utilización, además de cuantificar el valor de una innovación, para realizar comparaciones entre diferentes áreas de enfermedades y facilitar las decisiones, puesto que hay que considerar que la introducción de nuevos medicamentos pueden conducir a:

1. Un cambio en los patrones de tratamiento que podía compensar los costos más altos de los medicamen- tos con ahorros o beneficios sociales

2. Un aumento de los costos totales de atención médica, al mantener a los pacientes con vida y en tratamiento, basados en la evaluación de las necesidades de salud pública y estudios fármaco económicos

3. Un aumento de los costos totales, sin presentar resultados en términos de eficacia/ efectividad, seguridad y calidad de vida con aumentos significativos en el presupuesto, debido a la absorción de los costos de nuevos tratamientos, en ausencia de un proceso de selección y racionamiento estrictos.

Este último escenario en particular, más frecuente de lo esperado, no estaría promoviendo el aseguramiento del derecho a la salud para el resto de la población, puesto que la incorporación de estos nuevos medicamentos representarán varios cientos de millones de dólares adicionales por pocos pacientes, con pobres desenlaces la mayoría de las veces, que no inciden en mejorar la calidad de vida, tampoco en alcanzar la remisión de la enfermedad y si acaso en obtener po-

\footnotetext{
${ }^{11}$ Los programas de salud pública son aquellos que se ocupan de la atención de problemas que afectan a la colectividad y que por su alto impacto de contagio, complejidad diagnóstica o de tratamiento o magnitud en la carga de enfermedad, no pueden tratarse solo de manera individual, sino a través de una estrategia de control y prevención a nivel nacional y centralizada, en la mayoría de los casos necesariamente gubernamental. Entre los principales tenemos: Programa de inmunizaciones, VIH-SIDA, Tuberculosis, Malaria-Dengue, Leishmaniasis, Chagas.
} 
cos meses adicionales de vida a cambio de ingentes presupuestos que se recortan a la mayoría de la población poniendo en riesgo la sostenibilidad de todo el sistema sanitario.

Al analizar un grupo de medicamentos de alto impacto financiero que cubre mayoritariamente a enfermedades de baja prevalencia, raras y catastróficas, se puede apreciar que la incorporación indiscriminada de nuevas tecnologías sanitarias reduciría la brecha entre el presupuesto y el gasto en medicamentos, planteando serios problemas en el sistema de salud. Y a su vez, crearía una profunda desigualdad entre el gasto de medicamentos de alto impacto y el gasto en otros programas de salud pública.

Todo esto indica que la percepción de los tomadores de decisiones de aprobación de medicamentos con efica- cia marginal o nula es que, la salud que se espera obtener del uso de medicamentos de alto impacto supera la salud que se espera perder en otros lugares, por el desplazamiento de otras actividades de atención de la salud. El valor de medicamentos para la atención de pocos casos, mayoritariamente oncológicos, podría poner en riesgo el financiamiento de la mayoría de prestaciones o medicamentos para la mayoría de personas.

Ante lo mencionado el Gobierno deberá realizar una estimación empírica de los costos de oportunidad que enfrenta el Sistema Nacional de Salud, y ponderar qué política pública va a impulsar si los beneficios para la salud asociados con las nuevas tecnologías son mayores que la salud que probablemente se perderá en otras partes del sistema.

\section{CONCLUSIONES}

En la relación del financiamiento en Ecuador, Gobierno $51 \%$ y Hogares 49\%, estimada por la OMS en 2016, parece haber incentivos para acentuar el porcentaje de participación de estos últimos en los años venideros. Así, el bajo o negativo crecimiento de la economía previsto para 2019 y 2020, en aras del equilibrio fiscal y de no mediar un mayor entendimiento por parte del Gobierno sobre el rol de la salud en el crecimiento económico del país, estimulará posiblemente incluso un cambio en dicha relación, a favor de una todavía mayor proporción del aporte de los hogares en el financiamiento de la salud.

Aunque el rubro presupuestario de Talento Humano sigue siendo todavía el más importante, medicamentos e insumos médicos han incrementado de ma- 
nera notable su crecimiento, cambiando el relacionamiento tradicional, y poniendo severos retos en el ámbito financiero del sector salud; mismos que los administradores de recursos sanitarios deberán sortear. Actualmente, para la toma de decisiones es necesario tomar en cuenta además de los muchos aspectos financieros, los costos de oportunidad, y decidir si el reconocer las necesidades de unos cuantos es un argumento lo suficientemente sólido para justificar la afectación de la mayoría a quienes se les restringirá los programas de salud pública. Una mirada a los problemas que experimentan países hermanos que no pueden invertir en la protección sanitaria a través de programas colectivos, debería bastar para establecer los parámetros de conveniencia o no, para trasgredir umbrales de costo-efectividad que los países establecen para el uso de sus recursos.

La existencia de un financiamiento bicéfalo, con severos problemas de eficiencia en los subsistemas prestacionales de salud públicos, especialmente el de la seguridad social, sumado a la inexistencia de controles efectivos sistémicos en general, es insostenible y lleva al colapso generalizado de la prestación pública. De no instaurarse de manera urgente una instancia que pueda realizar el acompañamiento técnico de los subsistemas administradores de recursos para adquirir prestaciones de salud, y la po- sibilidad de controles técnicos efectivos en la gestión de los recursos, no cambiarán las racionalidades institucionales capturadas por grupos rentistas.

Finalmente, de lo analizado se desprende que es necesario definir un solo modelo de financiamiento, sea este basado en impuestos fiscales, financiado desde el Gobierno a través de impuestos, o sea este un esquema basado en un seguro social contributivo único, fincado en contribuciones personales, patronales y gubernamentales.

Desde una perspectiva técnica el mejor negocio para Ecuador, si quiere guardar progresividad en los aportes (quienes más tienen aportan más), rentabilidad de las inversiones realizadas (mayor inversión social y rentabilidad de costos hundidos), una burocracia profesional más eficiente y menos cara y una cultura institucional de carácter nacional; la balanza se inclina hacia la consolidación de un Sistema Nacional de Salud. Ello por supuesto no implica la eliminación de la complementariedad y acotación del sistema de provisión desde la seguridad social, así como el control técnico de todos los subsistemas de salud bajo los principios de un Sistema Nacional de Salud, para lo cual es necesario iniciar con la creación de una entidad concreta, con roles de orientación y control especializado específico. Considerar establecer un solo modelo público es quizás la medida que, 
políticamente fuerte, será la única que financiamiento del sector salud en Ecuapueda resolver el complejo problema de dor, presente y futuro. 


\section{REFERENCIAS BIBLIOGRÁFICAS}

Barro, R. (2013). No Title.

BCE. (n.d.). Inversión Pública. Quito Ecuador. Retrieved from www.bce. gob.ec

Carriel-Mancilla, J. (2012). Gasto Público en Salud en Ecuador. Rev. Med. FCM-UCSG, Año, 2018(1), 53-60. https://doi.org/ISSN - 1390-0218

Durán-Lima J, C. S. (2016). Estimación del empleo directo e indirecto asociado a las exportaciones del Ecuador a la Unión Europea (Comercio Internacional No. 127). Chile. Retrieved from https://www. google.com/search?q=por+cada+empleo+directo+cuantos+indirectos+se+generan\&ie $=$ utf-8\&oe $=$ utf-8\&client $=-$ firefox-b

Dussault, G., \& Dubois, C. A. (2003). Human resources for health policies: A critical component in health policies. Human Resources for Health, 1(May). https://doi. org/10.1186/1478-4491-1-1

Giovanella, L, Feo, O, Faria, M, Tobar, S. (2012). Sistema de Salud en Ecuador. Río de Janeiro: ISAGS-UNASUR. Retrieved from http://isags-unasur.org/es/sistema-de-salud-en-ecuador/

INEC. (2015). Ecuador: Proyección de Población 2010-2020. Quito Ecuador.
Retrieved from www.inec.gob.ec

INEC. (2018). ENCUESTA NACIONAL DE EMPLEO, DESEMPLEO Y SUBEMPLEO ENEMDU Indicadores Laborales Marzo 2018. Retrieved from https://www.google.com/ search?client $=$ firefox $-\mathrm{b} \& \mathrm{ei}=\mathrm{E}-$ jCNXI6MGtHc5gLO_6g4\&q=cual+es+la+poblacion+economicamente+activa+del+ecuador$+2018 \&$ oq $=$ cual + es + la + pea + ec uador+\&gs_l=psy-ab.1.3.0i22i30l 6.151798.156730..159976...0.0..0.1 82.3552.0j23..........1...gws-wiz....

Instituto Ecuatoriano de Estadísticas y Censos. (n.d.). Estadísticas de Salud. Retrieved from http://www. inec.gob.ec/estadisticas/?option=com_content $\&$ view $=$ article\&id $=77 \&$ ltemid $=49$

Kutzin, J. (2008). Health financing policy: a guide for decision-makers. Health Financing Policy Paper, Division of Country Health Systems.

Londoño, J., \& Frenk, J. (1997). Pluralismo estructurado: Hacia un modelo innovador para la reforma de los sistemas de salud en América Latina. Documento de Trabajo Del Departamento de Investigaciones Del Banco Interamericano de Desarrollo. Retrieved from https://www.econstor.eu/ 
handle/10419/87917

Lucio, R., Villacrés, N., \& Henríquez, R. (2011). [The health system of Ecuador]. Salud Pública de México, 53 Supp/ 2, s177-87. Retrieved from http://www.ncbi.nlm.nih.gov/ pubmed/21877083

Maceira, D. (2014). Cuadrantes de análisis en los sistemas de salud de América Latina. Documentos de Trabajo CEDES, 122/2014.

Miguel, M., \& Malo, N. (2014). Reforma de salud en Ecuador: nunca más el derecho a la salud como un privilegio. Revista Peruana de Medicina Experimentaly Salud Pública, 31(4), 754-761.

Ministerio de Finanzas. (n.d.). E-Sigef.

Ministerio de Salud Pública. Subsecretaría Nacional de Gobernanza de la Salud Pública. (2013). Manual del Modelo de Atención Integral de Salud (MAIS). (MSP, Ed.) (Primera Ed). Quito.

OMS. (n.d.). Portal de información estadistica y bases de datos en gastos en salud. Retrieved from http:// www.who.int/health-accounts/ ghed/en/

OMS. (2010). Informe sobre la salud en el mundo. La financiación de los sistemas de salud: el camino hacia la cobertura universal. Ginebra.

OPS/OMS. (2014). Estrategia para el acceso universal a la salud y la cobertu- ra universal de salud. CD53/5. Rev. 2. OPS/OMS.

OPS/OMS. (2015). Cómo tomar decisiones justas en el camino hacia la cobertura universal de salud.

OPS/OMS. (2017). Informe Especial - Revista Panamericana de la Salud Publica. 41.

OPS/OMS. (2018). Espacio fiscal para la salud en america latina y el caribe. Washington D.C.

OTHS Colombia. (2015). Guia metodológica del observatorio de talento humano en salud.

RISSALUD. (2017). Remuneraciones en el sector salud Estudio de caso sobre un grupo de países de América Latina yel Caribe.

Sojo, A. (2017). Protección social en América Latina: la desigualdad en el banquillo. (CEPAL, Ed.) (1 ra ed.). Santiago de Chile: CEPAL - Naciones Unidas.

Stiglitz, J. (2003). La economia del sector publico (Segunda). Antoni Bosch.

WHO. (2000). Informe sobre la salud en el mundo 2000.

Xu K, Saksena P, Jowett M, Indikadahena C, Kutzin J, E. D. E. the B. paper 19. G. O. 2010. (2010). thresholds of health expenditure for protection against financial risk. World Health Report 2010. Background Paper. 


\section{ANEXO}

\section{Talento Humano por Tipología}

\begin{tabular}{|c|c|}
\hline Hospitales & Talento Humano \\
\hline Hospital de Especialidades & 4105 \\
\hline HOSPITAL DE ESPECIALIDADES PORTOVIEJO & 372 \\
\hline HOSPITAL DE ESPECIALIDADES GENERAL ABEL GILBERT PONTÓN & 1697 \\
\hline HOSPITAL DE ESPECIALIDADES EUGENIO ESPEJO & 2036 \\
\hline Hospital Especializado & 7009 \\
\hline HOSPITAL UNIVERSITARIO DE GUAYAQUIL & 912 \\
\hline HOSPITAL PSIQUIÁTRICO JULIO ENDARA & 180 \\
\hline HOSPITAL PEDIÁTRICO BACA ORTIZ & 1226 \\
\hline HOSPITAL PEDIÁTRICO ALFONSO VILLAGOMEZ & 141 \\
\hline HOSPITAL MATILDE HIDALGO DE PROCEL & 552 \\
\hline HOSPITAL GINECO OBSTÉTRICO PEDIÁTRICO DE NUEVA AURORA LUZ ELENA ARISMENDI & 817 \\
\hline HOSPITAL GINECO OBSTÉTRICO ISIDRO AYORA & 878 \\
\hline HOSPITAL GERIÁTRICO DOCTOR BOLÍVAR ARGUELLO P & 73 \\
\hline HOSPITAL DE INFECTOLOGÍA JOSE D RODRIGUEZ MARIDUENA & 416 \\
\hline HOSPITAL DE ATENCIÓN INTEGRAL DEL ADULTO MAYOR & 233 \\
\hline HOSPITAL PEDIÁTRICO FRANCISCO ICAZA BUSTAMANTE & 1352 \\
\hline HOSPITAL OBSTÉTRICO ANGELA LOAYZA DE OLLAGE & 229 \\
\hline Hospital General & 19874 \\
\hline HOSPITAL PROVINCIAL PUYO & 376 \\
\hline HOSPITAL PROVINCIAL GENERAL TEÓFILO DÁVILA & 748 \\
\hline HOSPITAL PROVINCIAL GENERAL SAN VICENTE DE PAÚL & 671 \\
\hline HOSPITAL PROVINCIAL GENERAL PABLO ARTURO SUÁREZ & 1131 \\
\hline HOSPITAL PROVINCIAL GENERAL MARTÍN ICAZA & 341 \\
\hline HOSPITAL PROVINCIAL GENERAL LUIS G. DAVILA & 408 \\
\hline HOSPITAL PROVINCIAL GENERAL JULUIS DOEPFNER & 249 \\
\hline HOSPITAL PROVINCIAL GENERAL ISIDRO AYORA & 749 \\
\hline HOSPITAL PROVINCIAL GENERAL HOMERO CASTANIER & 544 \\
\hline HOSPITAL PROVINCIAL GENERAL DOCENTE VICENTE CORRAL MOSCOSO & 1001 \\
\hline HOSPITAL PROVINCIAL GENERAL DOCENTE DE RIOBAMBA & 721 \\
\hline HOSPITAL PROVINCIAL GENERAL DOCENTE DE AMBATO & 874 \\
\hline HOSPITAL PROVINCIAL GENERAL DELFINA TORRES DE CONCHA & 819 \\
\hline HOSPITAL PROVINCIAL GENERAL DE LATACUNGA & 884 \\
\hline HOSPITAL PROVINCIAL DR. MARCO VINICIO IZA & 539 \\
\hline HOSPITAL PROVINCIAL DOCENTE ALFREDO NOBOA MONTENEGRO & 339 \\
\hline HOSPITAL PROVINCIAL DE PORTOVIEJO & 839 \\
\hline HOSPITAL PROVINCIAL JOSE MARÍA VELASCO IBARRA DE TENA & 682 \\
\hline HOSPITAL OSKAR JANDL & 128 \\
\hline HOSPITAL LEÓN BECERRA DE MILAGRO & 314 \\
\hline HOSPITAL GENERAL SANTO DOMINGO & 213 \\
\hline HOSPITAL GENERAL RODRÍGUEZ ZAMBRANO DE MANTA & 704 \\
\hline HOSPITAL GENERAL MONTE SINAÍ & 610 \\
\hline HOSPITAL GENERAL GUASMO SUR & 1066 \\
\hline HOSPITAL GENERAL GENERAL MIGUEL ALCÍVAR DE BAHÍA & 304 \\
\hline HOSPITAL GENERAL DR. LIBORIO PANCHANA SOTOMAYOR & 516 \\
\hline HOSPITAL GENERAL DOCENTE DE CALDERÓN & 1087 \\
\hline HOSPITAL GENERAL DE MACAS & 335 \\
\hline HOSPITAL GENERAL DE JIPIJAPA & 197 \\
\hline HOSPITAL GENERAL DE CHONE & 443 \\
\hline HOSPITAL PROVINCIAL GENERAL ENRIQUE GARCÉS & 1211 \\
\hline HOSPITAL DR. GUSTAVO DOMÍNGUEZ Z. & 831 \\
\hline
\end{tabular}

Fuente: Distributivo del Personal Asistencial de los Hospitales 